\title{
An Interactive Method for Two-plants Production Inventory Control with Two-warehouse Facility under Imprecise Environment
}

\author{
Samar Hazari ${ }^{1}$ \\ Dept of Mathematics, \\ NIT, Durgapur-713209, \\ West Bengal, India.
}

\author{
*Jayanta kumar Dey ${ }^{2}$ \\ Dept of Mathematics, \\ Mahisadal Raj College-721628, \\ West Bengal, India.
}

\author{
K. Maity ${ }^{3}$ \\ Dept of Mathematics, \\ Mugberia Gangadhar Maha- \\ vidyalaya-721425, West Bengal.
}

\author{
Samarjit $\operatorname{Kar}^{1}$ \\ Dept of Mathematics, \\ NIT, Durgapur-713209, \\ West Bengal, India.
}

\begin{abstract}
This paper develops an integrated production inventory model with an aim to minimize the cost of production per unit of the product and maximization of profit without compromising the quality of the product. In this model we consider two plants, two secondary warehouses (SWs) and two showrooms (SRs) those are adjacent to the respective plants. For the purpose, we assume to be erected by a firm two plants: Plant-I and Plant-II. Plant-I is situated in an urban area with a lower production capacity in comparison to its product demand where the demand meets through SR-I. On the other hand, Plant-II is situated in a rural area with higher production capacity in comparison to its product demand where the demand meets through SR-II. The excess production in Plant-II meets the current market demand in the area of Plant-I. Here, demand is assumed to be stock dependent in both the showrooms (SR-I and SR-II). Average profit in the integrated model is calculated and global optimum is obtained through a descriptive-cum-analytical review. The inventory parameters are taken as fuzzy numbers. The fuzzy numbers are first transformed into corresponding interval numbers and then follow the interval mathematics, the objective function for average profit is converted into respective multi-objective functions. Furthermore, the objective functions are being maximized and solved for a Pareto-optimum solution by interactive fuzzy decision-making procedure. The model also illustrates graphically and numerically.
\end{abstract}

\section{General Terms:}

Two-plants, Two-warehouse,

\section{Keywords:}

Inventory, Two-plants, Two-warehouse, Stock dependent demand, Fuzzy inventory cost parameters, Interactive decision making method.

\section{INTRODUCTION}

The works of Urban[34], Pal et.al.[30], Giri et.al.[11], Padmanabhan and Vrat [28], Sarkar et al[32], Giri and Chaudhuri[12] and others were developed for a single warehouse under the basic assumption that the available warehouse has unlimited storage capacity. However, this assumption is not realistic. Any warehouse has fi- nite storage capacity. On the other hand, inventory management is generally attracted for large stock for several reasons - an attractive price discount for bulk purchase; the replenishment cost including transportation cost is higher than the inventory related cost; the demand of an item is very high and so on. Therefore, due to space limitation of showroom (SR), one (or sometimes more than one) warehouse(s)are hired on rental basis to store the excess items.

The secondary warehouse (SW) may be located away from or nearer to SR. The actual service to the customer is done at SR only. As the holding cost in SW is greater than in SR, the stocks of SW are emptied first transporting the stocks from SW to SR by continuous or bulk release patterns in order to reduce the holding cost. Hartely [17]first introduced the basic two warehouse problem in his book "Operations Research - A Managerial Emphasis". In his analysis, he ignored the cost of transportation for transferring the items from SW to SR and proposed a heuristic procedure for determining the optimal order quantity. After Hartely[17], a number of research papers have been published by the different authors. Among them, the works done by Sarma[33], Dave[8],Goswami and Chaudhuri[13], Pakkala and Achary[29], Bhunia and Maiti[3,5], Benkherouf[2], Zhou[37] and Kar et. al.[19] are worth mentioning. However, all these models were based on an assumption that the rented warehouse has unlimited (infinite) storage capacity. Chung and Huang[7]proposed a two-warehouse inventory model for deteriorating items under a permissible delay in payments, but they assumed that the deterioration rate of the two warehouses were the same. Dey et al.[10] considered a finite time horizon inventory problem for a deteriorating item having two separate warehouses with interval-valued lead-time under inflation and a time value of money. Niu and Xie[27] modified Pakkala and Achary's[29]model (last-in-first-out) where inventory in the SW was stored last but would be consumed before those in the SR. Hsieh et al.[18] developed a deterministic inventory model for deteriorating items with two warehouses allowing for shortages and assuming the inventory costs in the SW to be higher than those in the SR. Lee and Hsu [20] extended the Lee and Ma[21],'s model using an approach which permitted variable production cycle times instead of equal production cycle times. Chung et al.[6] extended a two-warehouse inventory model with an imperfect quality production processes. Liao and Huang[24] studied an order-level inventory model for deteriorating items with two-storage facilities and a permissible delay in payment. Hariga[16] proposed an EOQ 
model with multiple storage facilities where both owned and rented warehouses had limited stock capacity. Liang and Zhou[23] investigated a two-warehouse inventory model for deteriorating items under conditionally permissible delay in payments. They assumed the rented warehouse had higher unit holding costs than the own warehouse but offered better preservation resulting in a lower rate of deterioration for the goods than in the own warehouse. In reality there are many situations in business sector where the demand rate is not constant but varies. It may depend on time, initial or on hand inventory levels, selling price, advertisement expenditure, the frequency of advertisement etc. There are certain types of items (like consumer goods, fashionable items etc.) for which, according to market research, customers are motivated by the display of the items in the showrooms i.e., the demand rate is dependent on the displayed inventory level. For these items, the consumption goes up if the inventory level is high and vice versa. Many researchers like Levin et al.[22] have observed that the presence of greater quantity of the same item tends to attract more customers. This implies that holding higher inventory level will probably make the retailer sell more items. Under this situation, the demand rate should depend on the inventory level. Such type of demand was considered by Urban[34], Gupta and Vrat[15], Mondal and Phaujdar [25,26], Urban [34,35], Bhuina and Maiti[4] etc. Zhou and Yang[38] presented a two-warehouse inventory model with stock-level-dependent demand rate.

In this study, we considered a production inventory model of two production plants (plant-I and plant-II), two secondary warehouses (SW-I and SW-II) and two showroom-cum-retail outlets (SR-I and SR-II). Production plant-I is situated in an urban area where the density of population is considerably high and thereby there is a high market demand of the product produced in plant-I. The cost of the factors of production in plant-I viz., raw-materials, labour, power, water supply, rent of the factory building etc. is very high and thereby the cost of the production per unit of the output is also very high. In this plant, the rate of production is less in comparison to the market demand of the output produced in plant-I. The plant-I has been erected in the area concerned, where there is a scarcity of the factors of production albeit the locational and other advantages for industrial output are prevalent in the area concerned. In order to minimize the total cost of production per unit of the output produced in both the plants and at the same time to meet the high market demand of the product exits in the area of plant-I, another plant-II has been erected in a rural area where the factors of production required in plant-I is less costly albeit the market demand of the output in the area of plant-II is less than that of the market demand in the area of plant-I. So, the cost of production per unit of output produced in Plant-II is less than that of the cost of production per unit of output produced in plant-I. It is mentioned here that the quality standard of the products produced in both the plants are maintained.

There is a large volume of literature on the 'two warehouse inventory model'. The literature suggests that the holding cost of secondary warehouse per unit is more than that of the holding cost per unit of showroom cum-outlet. Furthermore, the literature suggests that the holding cost per unit in the secondary warehouse is high due to the preservation cost for maintaining the quality of the product and other costs related to handling large quantity of the product in the secondary warehouse. In the present model it is presumed that the holding cost of secondary warehouses is less than the holding cost of showrooms cum-outlets inasmuch as the nature of the output/product produced in both the plants are nondeteriorating and without having any preservation cost. But it is important to mention here that both the secondary warehouses are located adjacent to the corresponding showroom-cum-retail outlets and the transportation cost from both the warehouses to showrooms is insignificant, and hence avoided. Furthermore, the product stored in SW-II is transferred to SW-I through bulk-release-pattern and corresponding transportation cost between two warehouses has been taken into account in the model. This apart, output transferred from both the plants to the showrooms-cum-retail outlets in a continuous-release-pattern. The customer service made from the both the showrooms and the products of both the plants transformed on a regular basis / constantly from both the warehouses to both the showrooms to fulfil the demand (as the demand of the product is stock-dependent) even though the holding cost per unit is higher than in showrooms than that of warehouses.

Average profit of the integrated model has been calculated and global optimum was obtained analytically. Here inventory cost parameters are taken imprecise i.e, fuzzy in nature. The said parameters are expressed by fuzzy numbers, Which are then converted into appropriate interval numbers following Grzegorzewski[14] and using the concepts of interval arithmetic, we have constructed an equivalent multi-objective deterministic model corresponding to the original problem. This equivalent problem has been solved by using interactive fuzzy decision making procedure. The Optimum and Pareto-optimum solutions are derived by using a gradient non-linear optimization technique-Generalized Reduced Gradient (GRG) method.

\section{THE NEAREST INTERVAL APPROXIMATION OF A FUZZY NUMBER}

According to Grzegorzewski[14], the nearest interval approximation of a Triangular fuzzy number(TFN) $\tilde{A}=\left(a_{1}, a_{2}, a_{3}\right)$ is $\left(\frac{a_{1}+a_{2}}{2}, \frac{a_{2}+a_{3}}{2}\right)$ and the nearest interval approximation of a Parabolic fuzzy number(PFN) $\tilde{A}=\left(a_{1}, a_{2}, a_{3}\right)$ is $\left(\frac{2 a_{1}+a_{2}}{3}, \frac{a_{2}+2 a_{3}}{3}\right)$.

\section{ASSUMPTIONS AND NOTATIONS}

Following are the assumptions and notations to develope the model:

\subsection{Assumptions}

(i) Model is developed for single item product.

(ii) Lead time is negligible.

(iii) Demand of customers in the showrooms SR-I and SR-II is stock dependent.

(iv) Shortage are allowed only in SR-I.

(v) Idle costs are taken into account.

(vi) Production capacities of two plants are different.

\subsection{Notations}

$q_{1 s}(t)=$ Inventory level of SR-I at time $\mathrm{t}$.

$q_{1 r}(t)=$ Inventory level of SW-I at time t.

$q_{2 s}(t)=$ Inventory level of SR-II at time t.

$q_{2 r}(t)=$ Inventory level of SW-II at time $\mathrm{t}$.

$P_{1}=$ Constant production rate of plant-I

$\left(P_{1}<a_{1}, a_{1}>0\right)$.

$P_{2}=$ Constant production rate of plant-II.

$W_{1}=$ Capacity of SR-I. 
$W_{2}=$ Capacity of SR-II.

$D_{1}(t)=$ Demand rate of SR-I, where

$$
D_{1}(t)= \begin{cases}a_{1} & \text { when } 0 \leq t<t_{1} \\ a_{1}+b_{1} W_{1} & \text { when } t_{1} \leq t<t_{2}+k^{\prime} \\ a_{1}+b_{1} q_{1 s}(t) & \text { when } t_{2}+k^{\prime} \leq t \leq T_{p_{1}}\end{cases}
$$

$a_{1}, b_{1}$ are constant.

$D_{2}(t)=$ Demand rate of SR-II, where

$$
D_{2}(t)= \begin{cases}a_{2} & \text { when } 0 \leq t<t_{1}^{\prime} \\ a_{2}+b_{2} W_{2} & \text { when } t_{1}^{\prime} \leq t<t_{3} \\ a_{2}+b_{2} q_{2 s}(t) & \text { when } t_{3} \leq t \leq T_{p_{2}}\end{cases}
$$

$a_{2}, b_{2}$ are constant.

$S_{1}=$ Shortage amount in SR-I at time $t_{1}$.

$c_{1 s}=$ Shortage cost per unit per unit time in SR-I of plant-I.

$h_{1 s}=$ Holding cost per unit per unit time of SR-I.

$h_{1 r}=$ Holding cost per unit per unit time of SW-I.

$h_{2 s}=$ Holding cost per unit per unit time of SR-II.

$h_{2 r}=$ Holding cost per unit per unit time of SW-II.

$c_{p_{1}}=$ Production cost per unit item for plant-I.

$c_{p_{2}}=$ Production cost per unit item for plant-II.

$s_{1}=$ Selling price per unit item in SR-I.

$s_{2}=$ Selling price per unit item in SR-II.

$p_{1}^{\prime}=$ Transfer rate of produce items from SW-I to SR-I during

$\left(t_{1}, t_{p_{1}}\right)$.

$p_{1}^{\prime \prime}=$ Transfer rate of produce items from SW-I to SR-I during $\left(t_{p_{1}}, t_{2}+k^{\prime}\right)$.

$k=$ Time duration of shifting the amount $k p_{1}^{\prime}$ from SW-II to SW-I during $\left(t_{1}, t_{p_{1}}\right)$.

$k^{\prime}=$ Time duration of shifting the amount $k^{\prime} p_{1}^{\prime \prime}$ from SW-II to SW-I during $\left(t_{p_{1}}, t_{2}\right)$.

$t_{1}=$ Time where SR-I received of $\operatorname{amount}\left(S_{1}+W_{1}\right)$.

$t_{p_{1}}=$ Time where plant-I stop its production.

$t_{p_{2}}=$ Time where plant-II stop its production.

$T_{p_{1}}=$ Time where items are exhausted from SR-I.

$T_{p_{2}}=$ Time where items are exhausted from SR-II.

$t_{2}=$ Time of shifting last lot from SW-II to SW-I.

$t_{3}=$ Time where items are exhausted from SW-II.

$A_{1 s}=$ Ordering cost for SR-I.

$A_{2 s}=$ Ordering cost for SR-II.

$A_{1 r}=$ Ordering cost for SW-I.

$A_{2 r}=$ Ordering cost for SW-II.

$i d_{1 s}=$ Idle cost per unit time of SR-I.

$i d_{2 s}=$ Idle cost per unit time of SR-II.

$i d_{1 r}=$ Idle cost per unit time of SW-I.

$i d_{2 r}=$ Idle cost per unit time of SW-II.

$n=$ Number of times to shifting the amount $p_{1}^{\prime} k$ in each time from SW-II to SW-I during $\left[t_{1}, t_{p_{1}}\right]$.

$r=$ Number of times to shifting the amount $p_{1}^{\prime \prime} k^{\prime}$ in each time from SW-II to SW-I during $\left[t_{p_{1}}, t_{p_{2}}\right]$.

$m-r=$ Number of times to shifting the amount $p_{1}^{\prime \prime} k^{\prime}$ in each time from SW-II to SW-I during $\left[t_{p_{2}}, t_{2}\right]$.

\section{MODEL DESCRIPTION AND DIAGRAMMATIC REPRESENTATION}

The production plants (i.e plant-I and plant-II), starts their production at time $t=0$ and produced items are selling through the showrooms(i.e SR-I and SR-II). In plant-I, the production rate is lower than the demand rate of SR-I and thus shortages occurs at SR-I for time period $\left(0, t_{1}\right)$. In plant-II, the production rate is higher than demand rate of SR-II, so excess items are stored in the secondary ware house SW-II at the rate $P_{2}-D_{2}$ in continuous release pattern. At time $t_{1}$, for one time, the items of amount $\left(S_{1}+W_{1}+k p_{1}^{\prime}\right)$ are transferred from SW-II to fulfill the shortage amounts $S_{1}$ at SR-I, to filled up the showroom SR-I by the amount $W_{1}$ and rest amount $k p_{1}^{\prime}$ is stored in the secondary ware house SW-I. Items are then transferred from SW-I to SR-I in continuous release pattern at the rates $p_{1}^{\prime}$ and $p_{1}^{\prime \prime}$ during the period $\left(t_{1}, t_{p_{1}}\right)$ and $\left(t_{p_{1}}, t_{2}+k^{\prime}\right)$ respectively to filled-up showroom SR-I continuously, as the demand rate at SR-I is stock depended. The items are transferred from SW-II to SW-I in bulk release pattern of amount $k p_{1}^{\prime}$ with time interval $k$ and of amount $k^{\prime} p_{1}^{\prime \prime}$ with time interval $k^{\prime}$ for $\mathrm{n}$ and $\mathrm{m}$ times during the periods $\left(t_{1}, t_{p_{1}}\right)$ and $\left(t_{p_{1}}, t_{2}\right)$ respectively. The amount $k p_{1}^{\prime}$ is transferred $\mathrm{r}$ times among $\mathrm{m}$ times with time interval $k^{\prime}$ for the period $\left(t_{p_{1}}, t_{p_{2}}\right)$. As demand is stock dependent at SR-II, so the items are continuously transferred with rate $D_{2}$ to filled-up the showroom SR-II from the production plant-II and SW-II during $\left(0, t_{p_{2}}\right)$ and $\left(t_{p_{2}}, t_{3}\right)$ respectively.

Mathematical formulation for different showrooms and secondary warehouses are depicted in different sub-section. The block diagrams of production inventory model is given in Fig.-1.

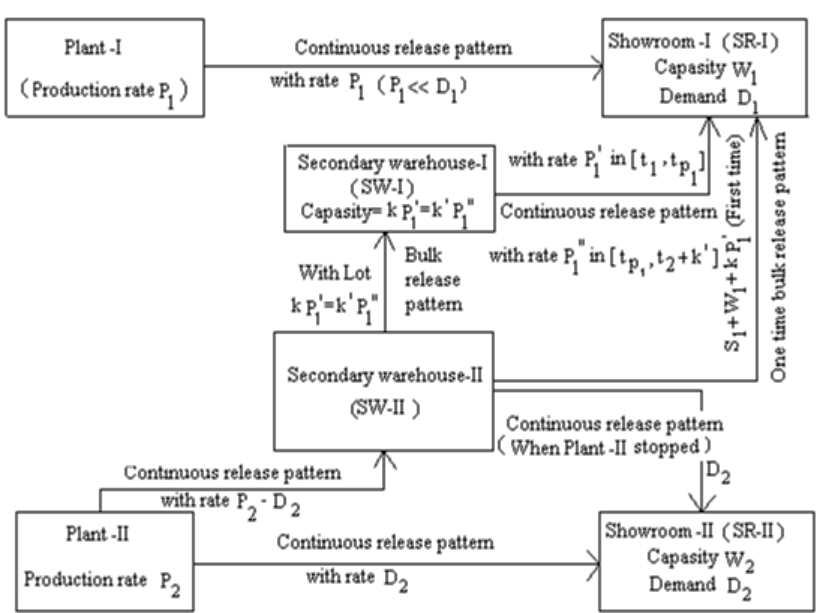

Fig. 1. Block diagram of the production inventory model 


\section{MATHEMATICAL FORMULATION OF THE MODEL}

\subsection{For Showroom-I(SR-I)}

Differential equation for the SR-I in $\left[0, T_{p_{1}}\right]$ is given by

$$
\frac{d q_{1 s}}{d t}=\left\{\begin{array}{lc}
P_{1}-a_{1}, & 0 \leq t<t_{1} \\
0, & t_{1} \leq t<t_{2}+k^{\prime} \\
-\left[a_{1}+b_{1} q_{1 s}(t)\right], & t_{2}+k^{\prime} \leq t \leq T_{p_{1}}
\end{array}\right.
$$

with boundary condition $q_{1 s}(t)=0$ at $t=0, T_{p_{1}}, q_{1 s}\left(t_{1}-0\right)=$

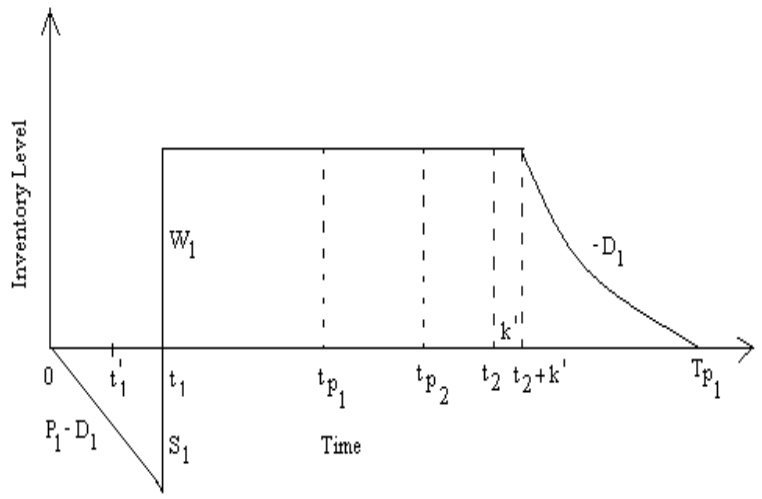

Fig. 2. Inventory level of SR-I

$-S_{1}$

and continuity condition $q_{1 s}(t)=W_{1}$ during $\left[t_{1}, t_{2}+k^{\prime}\right]$.

Solving the differential equation (1) with boundary conditions, we have

$$
q_{1 s}(t)= \begin{cases}\left(P_{1}-a_{1}\right) t, & 0 \leq t<t_{1} \\ W_{1}, & t_{1} \leq t<t_{2}+k^{\prime} \\ \frac{a_{1}}{b_{1}}\left(e^{b_{1}\left(T_{p_{1}}-t\right)}-1\right), & t_{2}+k^{\prime} \leq t \leq T_{p_{1}}\end{cases}
$$

Here $S_{1}=A+B k$ where A and B are given by

$$
A=\left(a_{1}-P_{1}\right)\left[\frac{t_{1}^{\prime}\left(P_{2}-\left(a_{2}+b_{2} W_{2}\right)\right)+W_{1}}{\left(P_{2}-\left(a_{2}+b_{2} W_{2}\right)\right)-\left(a_{1}-P_{1}\right)}\right]
$$

and, $\mathrm{B}=\left(a_{1}-P_{1}\right)\left[\frac{P_{1}^{\prime}}{\left(P_{2}-\left(a_{2}+b_{2} W_{2}\right)\right)-\left(a_{1}-P_{1}\right)}\right]$

Also, $P_{1}+p_{1}^{\prime}=a_{1}+b_{1} W_{1}, p_{1}^{\prime \prime}=a_{1}+b_{1} W_{1}$ and $T_{p_{1}}=t_{2}+k^{\prime}+\frac{1}{b_{1}} \log \left(1+\frac{b_{1} W_{1}}{a_{1}}\right)$

$S_{1 s}=$ Inventory Shortage cost of SR-I

$$
=c_{1 s}\left[-\int_{0}^{t_{1}}\left(P_{1}-a_{1}\right) t d t\right]=-c_{1 s}\left[\left(P_{1}-a_{1}\right) \frac{t_{1}^{2}}{2}\right]
$$

$H_{1 s}=$ Inventory holding cost $H_{1 s}$ of SR-I

$$
\begin{aligned}
& =h_{1 s}\left[W_{1}\left(t_{2}+k^{\prime}-t_{1}\right)+\int_{t_{2}+k^{\prime}}^{T_{p_{1}}} \frac{a_{1}}{b_{1}}\left(e^{b_{1}\left(T_{p_{1}}-t\right)}-1\right) d t\right] \\
& =h_{1 s}\left[W_{1}\left(t_{2}+k^{\prime}-t_{1}\right)+\frac{W_{1}}{b_{1}}-\frac{a_{1}}{b_{1}^{2}} \log \left(1+\frac{b_{1} W_{1}}{a_{1}}\right)\right]
\end{aligned}
$$

$I d_{1 s}=$ The idle cost of SR-I $=i d_{1 s}\left(T_{p_{2}}-T_{p_{1}}\right)$

\subsection{For Showroom-II (SR-II)}

Differential equation for the SR-II in $\left[0, T_{p_{2}}\right]$ is given by

$$
\frac{d q_{2 s}}{d t}=\left\{\begin{array}{lr}
P_{2}-\left(a_{2}+b_{2}+W_{2} q_{2 s}(t)\right), & 0 \leq t<t_{1}^{\prime} \\
0, & t_{1}^{\prime} \leq t<t_{3} \\
-\left(a_{2}+b_{2}+W_{2} q_{2 s}(t)\right), & t_{3} \leq t \leq T_{p_{2}}
\end{array}\right.
$$

with boundary condition $q_{2 s}(t)=0$, at $t=0, T_{p_{2}}$, and continuity

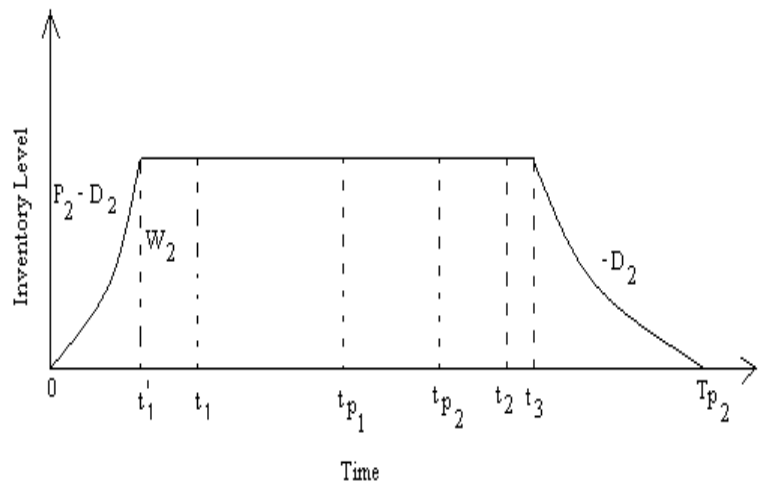

Fig. 3. Inventory level of SR-II

condition $q_{2 s}(t)=W_{2}$ in $\left[t_{1}^{\prime}, t_{3}\right]$.

Solving the differential equation(2) with boundary conditions, we have

$$
\begin{aligned}
& q_{2 s}(t)= \begin{cases}\frac{\left(P_{2}-a_{2}\right)}{b_{2}}\left(1-e^{-b_{2} t}\right), & 0 \leq t<t_{1}^{\prime} \\
W_{2}, & t_{1}^{\prime} \leq t<t_{3} \\
\frac{a_{2}}{b_{2}}\left(e^{b_{2}\left(T_{p_{2}}-t\right)}-1\right), & t_{3} \leq t \leq T_{p_{2}}\end{cases} \\
& \text { with } t_{1}^{\prime}=-\frac{1}{b_{2}} \log \left(1-\frac{b_{2} W_{2}}{a_{2}}\right) \\
& \text { and, } T_{p_{2}}=t_{3}+\frac{1}{b_{2}} \log \left(1+\frac{b_{2} W_{2}}{a_{2}}\right)=U+V k
\end{aligned}
$$

where $\mathrm{U}$ and $\mathrm{V}$ are given by

$$
\begin{aligned}
U= & \left(1+\frac{P_{2}}{a_{2}+b_{2} W_{2}}\right) \\
& {\left[\frac{\left(a_{1}-P_{1}\right)\left[\frac{t_{1}^{\prime}\left(P_{2}-\left(a_{2}+b_{2} W_{2}\right)\right)+W_{1}}{\left(P_{2}-\left(a_{2}+b_{2} W_{2}\right)\right)-\left(a_{1}-P_{1}\right)}\right]+W_{1}}{P_{2}-a_{2}-b_{2} W_{2}}\right] } \\
+ & \frac{1}{b_{2}} \log \left(1+\frac{b_{2} W_{2}}{a_{2}}\right)>0 \\
V= & \left(1+\frac{P_{2}}{a_{2}+b_{2} W_{2}}\right) \frac{p_{1}^{\prime}}{\left(P_{2}-a_{2}-b_{2} W_{2}\right)-\left(a_{1}-P_{1}\right)} \\
+ & n\left(\frac{P_{2}+a_{2}+b_{2} W_{2}}{a_{2}+b_{2} W_{2}}\right)+\frac{m\left(a_{2}+b_{2} W_{2}\right)+r P_{2}}{a_{2}+b_{2} W_{2}} \frac{p_{1}^{\prime}}{p_{1}^{\prime \prime}} \\
- & \frac{m+n}{a_{2}+b_{2} W_{2}} p_{1}^{\prime \prime} \\
H_{2 s}= & \text { Inventory holding cost of SR-II } \\
= & h_{2 s}\left[W_{1}\left(t_{3}-t_{1}\right)+\frac{\left(P_{2}-a_{2}\right)}{b_{2}} \int_{0}^{t_{1}^{\prime}}\left(1-e^{-b_{2} t}\right) d t\right.
\end{aligned}
$$




$$
\begin{aligned}
& \left.+\frac{a_{2}}{b_{2}} \int_{t_{3}}^{T_{p_{2}}}\left(e^{b_{2}\left(T_{p_{2}}-t\right)}-1\right) d t\right] \\
& =h_{2 s}\left[\frac{P_{2}-a_{2}}{b_{2}}\left[t_{1}^{\prime}+\frac{e^{-b_{2} t_{1}^{\prime}}}{b_{2}}\right] W_{2}\left(t_{3}-t_{1}\right)\right. \\
& \left.+\frac{W_{2}}{b_{2}}-\frac{a_{2}}{b_{2}^{2}} \log \left(1+\frac{b_{2} W_{2}}{a_{2}}\right)\right]
\end{aligned}
$$

\subsection{For Secondary Warehouse-I (SW-I)}

Differential equation for the SW-I in $\left[t_{1}, t_{2}+k^{\prime}\right]$ is given by

$$
\frac{d q_{1 r}}{d t}= \begin{cases}-p_{1}^{\prime}, & t_{1} \leq t<t_{p_{1}} \\ -p_{1}^{\prime \prime}, & t_{p_{1}} \leq t \leq t_{2}+k^{\prime}\end{cases}
$$

with boundary conditions

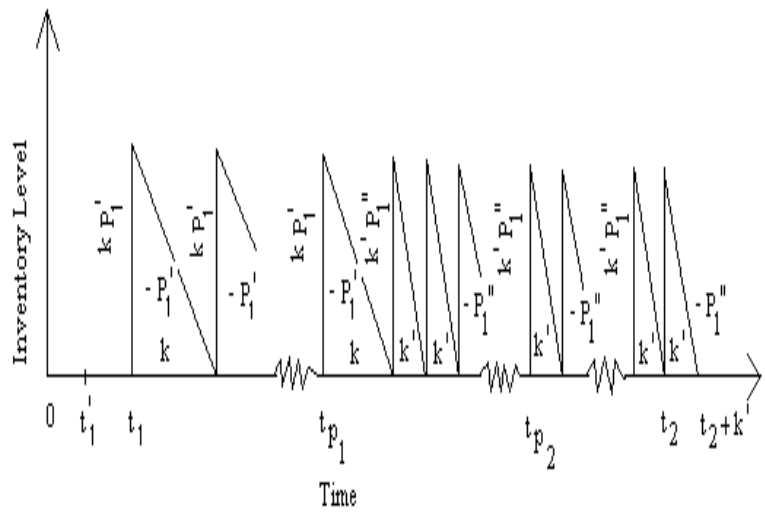

Fig. 4. Inventory level of SW-I

$$
q_{1 r}(t)=\left\{\begin{array}{l}
p_{1}^{\prime} k, \text { at } t=t_{1}+i k, i=0,1,2, ., ., n \\
0, \text { at } t=t_{1}+i k-0, i=0,1,2 ., ., ., n \\
p_{1}^{\prime \prime} k^{\prime}, \text { at } t=t_{p_{1}}+j k^{\prime}, j=0,1,2, ., ., ., m \\
0 \quad \text { at } t=t_{p_{1}}+j k^{\prime}-0, j=0,1,2,, ., ., m
\end{array}\right.
$$

where $t_{p_{1}}=t_{1}+n k, t_{2}=t_{p_{1}}+m k^{\prime}$ and $p_{1}^{\prime} k=p_{1}^{\prime \prime} k^{\prime}$.

Solving the differential equation(3) with boundary conditions, we have

$$
\begin{gathered}
q_{1 r}(t)=\left\{\begin{array}{c}
p_{1}^{\prime}\left[t_{1}+i k-t\right], t_{1}+(i-1) k \leq t<t_{1}+i k \\
i=1,2, ., ., ., n \\
p_{1}^{\prime \prime}\left[t_{p_{1}}+j k^{\prime}-t\right], t_{p_{1}}+(j-1) \leq t<t_{p_{1}}+j k^{\prime} \\
j=1,2, ., ., ., m \\
p_{1}^{\prime \prime}\left[k^{\prime}+t_{2}-t\right], t_{2} \leq t<t_{2}+k^{\prime}
\end{array}\right. \\
H_{1 r}=\text { Inventory holding cost of SW-I } \\
=h_{1 r}\left[\sum_{1}^{n} \int_{t_{1}+(i-1) k}^{t_{1}+i k} q_{1 r}(t) d t\right. \\
\left.+\sum_{1}^{m+1} \int_{t_{p_{1}}+(j-1) k^{\prime}}^{t_{p_{1}}+j k^{\prime}} q_{1 r}(t) d t\right]
\end{gathered}
$$

$$
\begin{aligned}
& =h_{1 r}\left[\frac{n}{2} p_{1}^{\prime} k^{2}+\frac{m+1}{2} p_{1}^{\prime \prime} k^{\prime 2}\right] \\
I d_{1 r} & =\text { The idle cost of SW-I } \\
& =i d_{1 r}\left(t_{1}+T_{p_{2}}-t_{2}-k^{\prime}\right)
\end{aligned}
$$

\subsection{For Secondary Warehouse-II (SW-II)}

Differential equation for the SW-II in $\left[0, t_{3}\right]$ is given by

$$
\frac{d q_{2 r}}{d t}=\left\{\begin{array}{l}
P_{2}-\left(a_{2}+b_{2} W_{2}\right), t_{1}^{\prime} \leq t<t_{p_{2}} \\
-\left(a_{2}+b_{2} W_{2}\right), t_{p_{2}} \leq t \leq t_{3}
\end{array}\right.
$$

with boundary conditions

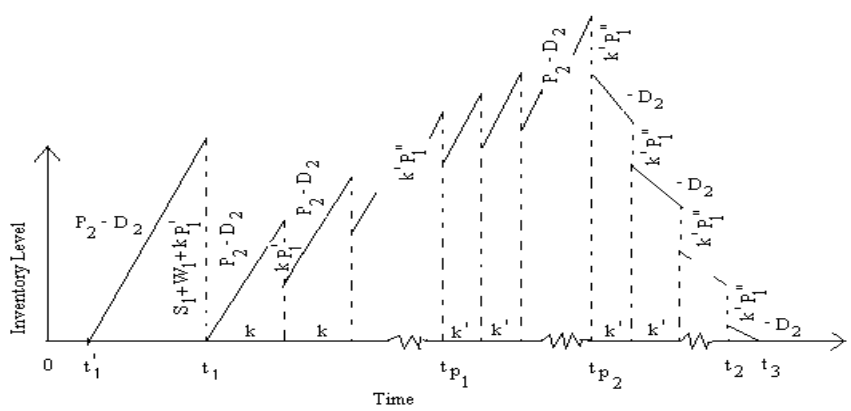

Fig. 5. Inventory level of SW-II

$$
q_{2 r}(t)=\left\{\begin{array}{l}
0, \quad \text { at } t=t_{1}^{\prime}, t_{1} \\
S_{1}+W_{1}+k p_{1}^{\prime}, \quad \text { at } t=t_{1}-0 \\
{\left[P_{2}-p_{1}^{\prime}-\left(a_{2}+b_{2} W_{2}\right)\right] i k,} \\
\text { at } t=t_{1}+i k, i=0,1,2 \ldots n \\
{\left[P_{2}-p_{1}^{\prime \prime}-\left(a_{2}+b_{2} W_{2}\right)\right] j k^{\prime},} \\
a t t=t_{p_{1}}+j k^{\prime}, \quad j=0,1,2 \ldots r \\
{\left[P_{2}-\left(a_{2}+b_{2} W_{2}\right)\right] t_{p_{2}}-j p_{1}^{\prime \prime} k^{\prime},} \\
a t t=t_{p_{1}}+j k^{\prime}, \quad \mathrm{J}=r+1, r+2 \ldots m \\
\quad \text { at } t=t_{3}
\end{array}\right.
$$

where $t_{p_{2}}=t_{p_{1}}+r k^{\prime}, t_{2}=t_{p_{1}}+m k^{\prime}$ and $t_{3}=t_{1}+n k+$ $m k^{\prime}+\frac{P_{2}}{a_{2}+b_{2} W_{2}}\left(t_{1}+n k+r k^{\prime}\right)-\frac{m+n}{a_{2}+b_{2} W_{2}} p_{1}^{\prime} k$

Solving the differential equation(4) with boundary conditions, we have

$q_{2 r}(t)=\left\{\begin{array}{l}{\left[P_{2}-\left(a_{2}+b_{2} W_{2}\right)\right]\left(t-t_{1}^{\prime}\right), t_{1}^{\prime} \leq t<t_{1}} \\ {\left[P_{2}-\left(a_{2}+b_{2} W_{2}\right)\right]\left(t-t_{1}^{\prime}\right)-(i-1) p_{1}^{\prime} k,} \\ t_{1}+(i-1) k \leq t<t_{1}+i k, i=1,2 \ldots n \\ {\left[P_{2}-\left(a_{2}+b_{2} W_{2}\right)\right]\left(t-t_{1}\right)-n k p_{1}^{\prime}-(j-1) p_{1}^{\prime \prime} k^{\prime},} \\ t_{p_{1}}+(j-1) k^{\prime} \leq t<t_{p_{1}}+j k^{\prime}, j=1,2 \ldots r \\ P_{2} t_{p_{2}}-\left(a_{2}+b_{2} W_{2}\right) t-(n-r) k p_{1}^{\prime}-(j-1) p_{1}^{\prime \prime} k^{\prime}, \\ t_{p_{2}}+(j-1) k^{\prime} \leq t<t_{p_{2}}+j k^{\prime}, j=1,2 \ldots m-r \\ \left(a_{2}+b_{2} W_{2}\right)\left(t_{3}-t\right), \quad t_{2} \leq t<t_{3}\end{array}\right.$

$H_{2 r}=$ Inventory holding cost of SW-II

$$
=h_{2 r}\left[\int_{t_{1}^{\prime}}^{t_{1}} q_{2 r}(t) d t+\sum_{1}^{n} \int_{t_{1}+(i-1) k}^{t_{1}+i k} q_{2 r}(t) d t\right.
$$




$$
\begin{aligned}
+ & \sum_{1}^{r} \int_{t_{p_{1}}+(j-1) k^{\prime}}^{t_{p_{1}}+j k^{\prime}} q_{2 r}(t) d t+\sum_{1}^{m-r} \int_{t_{p_{2}}+(j-1) k^{\prime}}^{t_{p_{2}}+j k^{\prime}} q_{2 r}(t) d t \\
+ & \left.\int_{t_{2}}^{t_{3}} q_{2 r}(t) d t\right] \\
& =h_{2 r}\left[\frac{P_{2}-\left(a_{2}-b_{2} W_{2}\right)}{2}\left(t_{1}-t_{1}^{\prime}\right)^{2}\right. \\
& +\frac{P_{2}-\left(a_{2}-b_{2} W_{2}\right)}{2} n^{2} k^{2}-\frac{n(n+1)}{2} p_{1}^{\prime} k^{2} \\
& +\frac{P_{2}-\left(a_{2}-b_{2} W_{2}\right)}{2}\left[2 n r k k^{\prime}+r^{2} k^{\prime 2}\right]-n r p_{1}^{\prime \prime} k^{\prime 2} \\
& -\frac{r(r+1)}{2} p_{1}^{\prime \prime} k^{\prime 2}+n P_{2}\left(t_{1}+n k+r k^{\prime}\right) k^{\prime} \\
& -\frac{\left(a_{2}-b_{2} W_{2}\right)}{2}\left[(m-r)^{2} k^{2}+2(m-r)\left(t_{1}+n k+r k^{\prime}\right) k^{\prime}\right] \\
& \left.-\frac{(m-r)(m+n-1)}{2} p_{1}^{\prime} k^{2}+\frac{\left(a_{2}-b_{2} W_{2}\right)}{2}\left(t_{2}-t_{3}\right)^{2}\right] \\
I d_{2 r} & =\text { The idle cost of SW-II }=i d_{2 r}\left(T_{p_{2}}-t_{3}\right) \\
C_{t} & =\text { total transportation cost } \\
& =c_{t}\left[S_{1}+W_{1}+(m+n+1) k p_{1}^{\prime}\right]
\end{aligned}
$$

\section{INTEGRATED MODEL:}

\subsection{In Crisp Environment}

Total profit of the production inventory system $T P$ is given by

$$
\begin{aligned}
& T P=\text { Total profit of the production inventory system } \\
& =\text { Revenue from sales -production cost }-S_{1 s}-H_{1 s}-H_{2 s} \\
& -H_{1 r}-H_{2 r}-I d_{1 s}-I d_{2 s}-I d_{1 r}-I d_{2 r}-C_{t}-A_{1 s}-A_{2 s}-A_{1 r}-A_{2 r} \\
& =X k^{2}+Y k+Z \text { where } \mathrm{X}, \mathrm{Y} \text { and } \mathrm{Z} \text { are given by } \\
& X=-\left[c_{1 s}\left(a_{1}-p_{1}\right)\left[\frac{B p_{1}^{\prime}}{\left(P_{2}-a_{2}-b_{2} W_{2}\right)^{2}}\right] \frac{p_{1}^{\prime}}{P_{2}-a_{2}-b_{2} W_{2}}\right. \\
& +c_{1 s}\left(a_{1}-p_{1}\right)\left[\frac{B^{2}}{\left(P_{2}-a_{2}-b_{2} W_{2}\right)^{2}}\right]+h_{1 r} \frac{B}{P_{2}-a_{2}-b_{2} W_{2}} \\
& {\left[n P_{2} \frac{p_{1}^{\prime}}{p_{1}^{\prime \prime}}-\left(a_{2}+b_{2} W_{2}\right)(m-r)\right]+h_{1 r}\left[\frac{n}{2} p_{1}^{\prime}+\frac{m+1}{2} \frac{p_{1}^{\prime 2}}{p_{1}^{\prime \prime}}\right]} \\
& +h_{2 r}\left[\frac{p_{1}^{\prime 2}}{2\left(P_{2}-a_{2}-b_{2} W_{2}\right)}+\frac{P_{2}-a_{2}-b_{2} W_{2}}{2}\left(n^{2}+r^{2}\right.\right. \\
& \left.+\frac{2 n r p_{1}^{\prime}}{p_{1}^{\prime}}\right)-\frac{n(n-1)}{2} p_{1}^{\prime}-r(n+r-1) \frac{p_{1}^{\prime 2}}{p_{1}^{\prime \prime}}+n P_{2}\left(n \frac{p_{1}^{\prime}}{p_{1}^{\prime \prime}}\right. \\
& \left.+r \frac{p_{1}^{\prime 2}}{p_{1}^{\prime \prime 2}}\right)-\frac{a_{2}+b_{2} W_{2}}{2}\left((m-r)^{2}+2(m-r)\left(n \frac{p_{1}^{\prime}}{p_{1}^{\prime \prime}}+r \frac{p_{1}^{\prime 2}}{p_{1}^{\prime \prime 2}}\right)\right. \\
& \left.-\frac{(m-r)(m+n-1)}{2} p_{1}^{\prime 2}\right) \frac{p_{1}^{\prime}}{P_{2}-a_{2}-b_{2} W_{2}}\left(n P_{2} \frac{p_{1}^{\prime}}{p_{1}^{\prime \prime}}\right. \\
& \left.\left.\left.-\left(a_{2}+b_{2} W_{2}\right)(m-r)\right)\right]+c_{s_{1}} \frac{\left(a_{1}-P_{1}\right) p_{1}^{\prime 2}}{2\left(P_{2}-a_{2}-b_{2} W_{2}\right)^{2}}\right]<0, \\
& Y=\left(s_{1}-s_{2}\right) B+\left[\frac{B}{P_{2}-a_{2}-b_{2} W_{2}}\right] \\
& {\left[\left(s_{1}-c_{p_{1}}\right) P_{1}+\left(s_{2}-c_{p_{2}}\right) P_{2}\right]}
\end{aligned}
$$

$-2 c_{1 s}\left(a_{1}-P_{1}\right) B\left[\left(P_{2}-a_{2}-b_{2} W_{2}\right) t_{1}^{\prime}\right.$

$\left.+\frac{A+W_{1}}{\left(P_{2}-a_{2}-b_{2} W_{2}\right)^{2}}\right]+\left(n+\frac{p_{1}^{\prime}}{P_{2}-a_{2}-b_{2} W_{2}}\right)$

$\left[\left(s_{1}-c_{p_{1}}\right) P_{1}+\left(s_{2}-c_{p_{2}}\right) P_{2}\right]+r\left(s_{2}-c_{p_{1}}\right)$

$P_{2} \frac{p_{1}^{\prime}}{p_{1}^{\prime \prime}}+\left(s_{1}-s_{2}\right)(m+n+1) p_{1}^{\prime}$

$-c_{s_{1}}\left(a_{1}-p_{1}\right)\left[t_{1}^{\prime}+\frac{A+W_{1}}{P_{2}-a_{2}-b_{2} W_{2}}\right]$

$\frac{p_{1}^{\prime}}{P_{2}-a_{2}-b_{2} W_{2}}-h_{1 s} W_{1}\left[(n+1)+\frac{m p_{1}^{\prime}}{p_{1}^{\prime \prime}}\right]$

$-h_{2 s}\left[W_{2}\left(1+\frac{P_{2}}{a_{2}-b_{2} W_{2}}\right)\left(\frac{B}{P_{2}-a_{2}-b_{2} W_{2}}\right)\right.$

$\left.-\frac{a_{2}}{b_{2}^{2}} \log \left(1+\frac{b_{2} W_{2}}{a_{2}}\right)\right]-h_{2 s} W_{2}[n$

$+\frac{p_{1}^{\prime}}{P_{2}-a_{2}-b_{2} W_{2}}+\frac{m p_{1}^{\prime}}{p_{1}^{\prime \prime}}+\frac{P_{2}}{a_{2}+b_{2} W_{2}}$

$\left.\left(n+\frac{p_{1}^{\prime}}{P_{2}-a_{2}-b_{2} W_{2}}+\frac{r p_{1}^{\prime}}{p_{1}^{\prime \prime}}\right)-\frac{(m+n) p_{1}^{\prime}}{a_{2}-b_{2} W_{2}}\right]$

$-h_{1 r}\left(t_{1}^{\prime}+\frac{A+W_{1}}{P_{2}-a_{2}-b_{2} W_{2}}\right)\left[n P_{2} \frac{p_{1}^{\prime}}{p_{1}^{\prime \prime}}-\left(a_{2}+b_{2} W_{2}\right)\right.$

$$
(m-r)]-h_{2 r} \frac{P_{2}}{2}\left[n+\frac{p_{1}^{\prime}}{P_{2}-a_{2}-b_{2} W_{2}}+\frac{r p_{1}^{\prime}}{p_{1}^{\prime \prime}}\right.
$$$$
\left.-\frac{m+n}{2} p_{1}^{\prime}\right]-i d_{1 s} \frac{r p_{1}^{\prime}}{p_{1}^{\prime \prime}}-\left(i d_{1 r}+h_{2 r} \frac{P_{2}}{2}\right)
$$$$
\left(\frac{B}{P_{2}-a_{2}-b_{2} W_{2}}\right)-i d_{1 r}\left[\frac{p_{1}^{\prime}}{P_{2}-a_{2}-b_{2} W_{2}}\right.
$$$$
\left.-\frac{(m-r+1) p_{1}^{\prime}}{p_{1}^{\prime \prime}}\right]-c_{t}(m+m+1) p_{1}^{\prime}
$$

$$
\begin{aligned}
\text { and, } \mathrm{Z}= & \left(s_{1}-s_{2}\right)\left(A+W_{1}\right)+\left[t_{1}^{\prime}+\frac{A+W_{1}}{P_{2}-a_{2}-b_{2} W_{2}}\right] \\
& {\left[\left(s_{1}-c_{p_{1}}\right) P_{1}+\left(s_{2}-c_{p_{2}}\right) P_{2}\right]-c_{1 s}\left(a_{1}-P_{1}\right) } \\
& {\left[t_{1}^{\prime}+\frac{A+W_{1}}{P_{2}-a_{2}-b_{2} W_{2}}\right]^{2}-c_{1 s}\left(a_{1}-P_{1}\right) } \\
& {\left[\frac{\left(P_{2}-a_{2}-b_{2} W_{2}\right) t_{1}^{\prime}+A+W_{1}}{P_{2}-a_{2}-b_{2} W_{2}}\right]^{2} } \\
- & h_{1 s}\left[\frac{W_{1}}{b_{1}}-\frac{a_{1}}{b_{1}^{2}} \log \left(1+\frac{b_{1} W_{1}}{a_{1}}\right)\right] \\
- & h_{2 s}\left[\frac{P_{2}-a_{2}}{b_{2}}\left(t_{1}^{\prime}+\frac{1}{b_{2}} \frac{P_{2}-a_{2}-b_{2} W_{2}}{P_{2}-a_{2}}\right)\right. \\
+ & W_{2}\left(1+\frac{P_{2}}{a_{2}-b_{2} W_{2}}\right)\left(t_{1}^{\prime}+\frac{A+W_{1}}{P_{2}-a_{2}-b_{2} W_{2}}\right) \\
- & \left.\frac{a_{2}}{b_{2}^{2}} \log \left(1+\frac{b_{2} W_{2}}{a_{2}}\right)\right]-\left(i d_{1 r}+h_{2 r} \frac{P_{2}}{2}\right) \\
& \left(t_{1}^{\prime}+\frac{A+W_{1}}{P_{2}-a_{2}-b_{2} W_{2}}\right)-i d_{2 r}\left[\frac{1}{b_{2}} \log \left(1+\frac{b_{2} W_{2}}{a_{2}}\right)\right. \\
- & \left.c_{t}\left(A+W_{1}\right)-A_{1 s}-A_{2 s}-A_{1 r}-A_{2 r}\right]
\end{aligned}
$$


The average profit $\mathrm{ATP}(\mathrm{k})$ is given by

$$
A T P(k)=\frac{T P}{T_{p_{2}}}=\frac{X k^{2}+Y k+Z}{U+V k}
$$

For optimum value of $\operatorname{ATP}(\mathrm{k})$,we must have

$$
\begin{gathered}
\frac{d(A T P)}{d k}=0 \\
\Rightarrow \quad V X k^{2}+2 U X k+(U Y-V Z)=0 \\
\Rightarrow \quad k=-\frac{U}{V} \pm \sqrt{\frac{U^{2}}{V^{2}}-\frac{U Y-V Z}{V X}} \\
\text { Now } \left.\frac{d^{2}(A T P)}{d k^{2}}\right]_{k=k^{*}}<0 \\
\text { for } U^{2} X-V(U Y-V Z)<0 \\
\text { and } U Y-V Z>0
\end{gathered}
$$

Where $k^{*}$ is the corresponding optimal value of $\mathrm{k}$ and given by

$$
k^{*}=-\frac{U}{V}+\sqrt{\frac{U^{2}}{V^{2}}-\frac{U Y-V Z}{V X}}
$$

Therefore ATP is concave for $k=k^{*}$ if the inequations in (6) holds. Substituting the value of $k^{*}$ in (5) we get the optimum value $A T P^{*}(k)$ of $A T P(k)$.

\subsection{In Fuzzy Environment}

Considering all holding costs, shortage costs, set-up costs, and transportation cost as fuzzy numbers and then from equation (5), average total profit of the system is given by

$$
A \tilde{T} P(k)=\frac{T P}{T_{p_{2}}}=\frac{\tilde{X} k^{2}+\tilde{Y} k+\tilde{Y}}{U+V k}
$$

where $\tilde{X}, \tilde{Y}$ and $\tilde{Z}$ are given by

$$
\begin{aligned}
\tilde{X} & =-\left[\tilde{c_{1}}\left(a_{1}-p_{1}\right)\left[\frac{B p_{1}^{\prime}}{\left(P_{2}-a_{2}-b_{2} W_{2}\right)^{2}}\right] \frac{p_{1}^{\prime}}{P_{2}-a_{2}-b_{2} W_{2}}\right. \\
& +\tilde{c_{1}}\left(a_{1}-p_{1}\right)\left[\frac{B^{2}}{\left(P_{2}-a_{2}-b_{2} W_{2}\right)^{2}}\right]+\tilde{h_{1 r}} \frac{B}{P_{2}-a_{2}-b_{2} W_{2}} \\
& {\left[n P_{2} \frac{p_{1}^{\prime}}{p_{1}^{\prime \prime}}-\left(a_{2}+b_{2} W_{2}\right)(m-r)\right]+\tilde{h_{1}}\left[\frac{n}{2} p_{1}^{\prime}+\frac{m+1}{2} \frac{p_{1}^{\prime 2}}{p_{1}^{\prime \prime}}\right] } \\
& +\tilde{h_{2 r}}\left[\frac{p_{1}^{\prime 2}}{2\left(P_{2}-a_{2}-b_{2} W_{2}\right)}+\frac{P_{2}-a_{2}-b_{2} W_{2}}{2}\left(n^{2}+r^{2}\right.\right. \\
& \left.+\frac{2 n r p_{1}^{\prime}}{p_{1}^{\prime}}\right)-\frac{n(n-1)}{2} p_{1}^{\prime}-r(n+r-1) \frac{p_{1}^{\prime 2}}{p_{1}^{\prime \prime}}+n P_{2}\left(n \frac{p_{1}^{\prime}}{p_{1}^{\prime \prime}}\right. \\
& \left.+r \frac{p_{1}^{\prime 2}}{p_{1}^{\prime \prime 2}}\right)-\frac{a_{2}+b_{2} W_{2}}{2}\left((m-r)^{2}+2(m-r)\left(n \frac{p_{1}^{\prime}}{p_{1}^{\prime \prime}}+r \frac{p_{1}^{\prime 2}}{p_{1}^{\prime 2}}\right)\right. \\
& \left.-\frac{(m-r)(m+n-1)}{2} p_{1}^{\prime 2}\right) \frac{p_{1}^{\prime}}{P_{2}-a_{2}-b_{2} W_{2}}\left(n P_{2} \frac{p_{1}^{\prime}}{p_{1}^{\prime \prime}}\right. \\
& \left.\left.\left.-\left(a_{2}+b_{2} W_{2}\right)(m-r)\right)\right]+\tilde{c_{1 s}} \frac{\left(a_{1}-P_{1}\right) p_{1}^{\prime 2}}{2\left(P_{2}-a_{2}-b_{2} W_{2}\right)^{2}}\right] \\
\tilde{Y} & =\left(s_{1}-s_{2}\right) B+\left[\frac{B}{P_{2}-a_{2}-b_{2} W_{2}}\right]\left[\left(s_{1}-c_{p_{1}}\right) P_{1}\right. \\
& \left.+\left(s_{2}-c_{p_{2}}\right) P_{2}\right]-2 \tilde{c_{1}}\left(a_{1}-P_{1}\right) B\left[\left(P_{2}-a_{2}-b_{2} W_{2}\right) t_{1}^{\prime}\right.
\end{aligned}
$$

$$
\begin{aligned}
& \left.+\frac{A+W_{1}}{\left(P_{2}-a_{2}-b_{2} W_{2}\right)^{2}}\right]+\left(n+\frac{p_{1}^{\prime}}{P_{2}-a_{2}-b_{2} W_{2}}\right)\left[\left(s_{1}-c_{p_{1}}\right) P_{1}\right. \\
& \left.+\left(s_{2}-c_{p_{2}}\right) P_{2}\right]+r\left(s_{2}-c_{p_{1}}\right) P_{2} \frac{p_{1}^{\prime}}{p_{1}^{\prime \prime}}+\left(s_{1}-s_{2}\right) \\
& (m+n+1) p_{1}^{\prime}-\tilde{c_{1 s}}\left(a_{1}-p_{1}\right)\left[t_{1}^{\prime}\right. \\
& \left.+\frac{A+W_{1}}{P_{2}-a_{2}-b_{2} W_{2}}\right] \frac{p_{1}^{\prime}}{P_{2}-a_{2}-b_{2} W_{2}} \\
& -\tilde{h_{1 s}} W_{1}\left[(n+1)+\frac{m p_{1}^{\prime}}{p_{1}^{\prime \prime}}\right]-\tilde{h_{2 s}}\left[W_{2}(1\right. \\
& \left.+\frac{P_{2}}{a_{2}-b_{2} W_{2}}\right)\left(\frac{B}{P_{2}-a_{2}-b_{2} W_{2}}\right)-\frac{a_{2}}{b_{2}^{2}} \log (1+ \\
& \left.\left.\frac{b_{2} W_{2}}{a_{2}}\right)\right]-\tilde{h_{2 s}} W_{2}\left[n+\frac{p_{1}^{\prime}}{P_{2}-a_{2}-b_{2} W_{2}}+\frac{m p_{1}^{\prime}}{p_{1}^{\prime \prime}}\right. \\
& +\frac{P_{2}}{a_{2}+b_{2} W_{2}}\left(n+\frac{p_{1}^{\prime}}{p_{2}-a_{2}-b_{2} W_{2}}+\frac{r p_{1}^{\prime}}{p_{1}^{\prime \prime}}\right) \\
& \left.-\frac{(m+n) p_{1}^{\prime}}{a_{2}-b_{2} W_{2}}\right]-\tilde{h_{1 r}}\left(t_{1}^{\prime}+\frac{A+W_{1}}{P_{2}-a_{2}-b_{2} W_{2}}\right) \\
& {\left[n P_{2} \frac{p_{1}^{\prime}}{p_{1}^{\prime \prime}}-\left(a_{2}+b_{2} W_{2}\right)(m-r)\right]-\tilde{h_{2 r}} \frac{P_{2}}{2}[n+} \\
& \left.\frac{p_{1}^{\prime}}{P_{2}-a_{2}-b_{2} W_{2}}+\frac{r p_{1}^{\prime}}{P_{1}^{\prime \prime}}-\frac{m+n}{2} p_{1}^{\prime}\right]-i \tilde{d}_{1 s} \frac{r p_{1}^{\prime}}{p_{1}^{\prime \prime}} \\
& -\left(i \tilde{d}_{1 r}+\tilde{h_{2 r}} \frac{P_{2}}{2}\right)\left(\frac{B}{P_{2}-a_{2}-b_{2} W_{2}}\right) \\
& -i \tilde{d}_{1 r}\left[\frac{p_{1}^{\prime}}{P_{2}-a_{2}-b_{2} W_{2}}-\frac{(m-r+1) p_{1}^{\prime}}{p_{1}^{\prime \prime}}\right] \\
& -\tilde{c_{t}}(m+m+1) p_{1}^{\prime}
\end{aligned}
$$

and, $\tilde{Z}=\left(s_{1}-s_{2}\right)\left(A+W_{1}\right)+\left[t_{1}^{\prime}+\frac{A+W_{1}}{P_{2}-a_{2}-b_{2} W_{2}}\right]$

$\left[\left(s_{1}-c_{p_{1}}\right) P_{1}+\left(s_{2}-c_{p_{2}}\right) P_{2}\right]-\tilde{c}_{1 s}\left(a_{1}-P_{1}\right)$

$\left[t_{1}^{\prime}+\frac{A+W_{1}}{P_{2}-a_{2}-b_{2} W_{2}}\right]^{2}-\tilde{c_{1 s}}\left(a_{1}-P_{1}\right)$

$\left[\frac{\left(P_{2}-a_{2}-b_{2} W_{2}\right) t_{1}^{\prime}+A+W_{1}}{P_{2}-a_{2}-b_{2} W_{2}}\right]^{2}$

$-\tilde{h_{1 s}}\left[\frac{W_{1}}{b_{1}}-\frac{a_{1}}{b_{1}^{2}} \log \left(1+\frac{b_{1} W_{1}}{a_{1}}\right)\right]-\tilde{h_{2 s}}\left[\frac{P_{2}-a_{2}}{b_{2}}\right.$

$\left(t_{1}^{\prime}+\frac{1}{b_{2}} \frac{P_{2}-a_{2}-b_{2} W_{2}}{P_{2}-a_{2}}\right)+W_{2}(1+$

$\left.\frac{P_{2}}{a_{2}-b_{2} W_{2}}\right)\left(t_{1}^{\prime}+\frac{A+W_{1}}{P_{2}-a_{2}-b_{2} W_{2}}\right)$

$\left.-\frac{a_{2}}{b_{2}^{2}} \log \left(1+\frac{b_{2} W_{2}}{a_{2}}\right)\right]-\left(i \tilde{d}_{1 r}+\tilde{h_{2 r}} \frac{P_{2}}{2}\right)$

$\left(t_{1}^{\prime}+\frac{A+W_{1}}{P_{2}-a_{2}-b_{2} W_{2}}\right)-i \tilde{d}_{2 r}\left[\frac{1}{b_{2}} \log (1+\right.$

$\left.\frac{b_{2} W_{2}}{a_{2}}\right)-\tilde{c_{t}}\left(A+W_{1}\right)-\tilde{A_{1 s}}-\tilde{A_{2 s}}$

$\left.-\tilde{A_{1 r}}-\tilde{A_{2 r}}\right]$

6.2.1 Deterministic representation of the proposed model. Following Grzegorzewski[14], the fuzzy numbers are now trans- 
formed to interval numbers and the expression (8) is expressed as

$$
A \tilde{T} P(k)=\left[F_{L}, F_{R}\right]
$$

(For detail calculations of $F_{L}$ and $F_{R}$, see Appendix- $A$ ) According to Dey et.al[10], the interval problem (9) can be represented as

$$
\operatorname{Maximize}\left\{F_{L}, F_{C}\right\}
$$

where $F_{C}=\left(F_{L}+F_{R}\right) / 2$.

\section{INTERACTIVE APPROACH}

Now considering the imprecise nature of DM's judgement, DM may have different fuzzy or imprecise goals for each of the objective functions and hence interactive approach is used for the man-machine interaction. To derive the membership functions $\mu_{F_{L}}$ and $\mu_{F_{C}}$ for the objective functions $F_{L}$ and $F_{C}$ respectively from DM's viewpoint, we first calculate individual minimum(i.e. $F_{L}^{\min }, F_{C}^{\min }$ ) and individual maximum(i.e. $F_{L}^{\max }, F_{C}^{\max }$ ) by a non-linear optimization technique. With the help of individual minimum and maximum, the DM can select any one from among the following three types of membership functions

(i) Linear membership functions.

(ii) Quadratic membership functions.

(ii) Exponential membership functions.

The membership functions $\mu_{F_{L}}$ and $\mu_{F_{C}}$ for the corresponding objective functions $F_{L}$ and $F_{C}$ can be written as

$$
\mu_{F_{k}}=\left\{\begin{array}{lll}
1, & \text { if } \quad F_{k} \leq F_{k}^{0}, \\
d_{k}, & \text { if } \quad F_{k}^{0} \leq F_{k} \leq F_{k}^{1}, \\
0, & \text { if } \quad F_{k}^{1} \leq F_{k}
\end{array}\right.
$$

where $F_{k}^{0}$ and $F_{k}^{1}$ are to be chosen such that $F_{k}^{\text {min }} \leq F_{k}^{0} \leq F_{k}^{1} \leq$ $F_{k}^{\max }$ and $d_{k}$ is a strictly monotonic decreasing continuous function of $F_{k}$ which may be linear or non-linear.

\subsection{Description of the Membership functions}

7.1.1 Linear membership function (Type-I). For each objective function, the corresponding Linear membership functions are as follows:

$$
\mu_{F_{k}}=\left\{\begin{array}{lrl}
1, & \text { if } \quad F_{k} \leq F_{k}^{0}, \\
1-\frac{F_{k}^{1}-F_{k}}{P_{k}}, & \text { if } \quad F_{k}^{0} \leq F_{k} \leq F_{k}^{1}, \\
0 & \text { if } \quad F_{k}^{1} \leq F_{k}
\end{array}\right.
$$

where $F_{k}^{0}$ and $F_{k}^{1}$ are to be chosen such that $F_{k}^{\text {min }} \leq F_{k}^{0} \leq F_{k}^{1} \leq$ $F_{k}^{\max }$ and $P_{k}=F_{k}^{1}-F_{k}^{0}$ is the tolerance of k-th objective function $F_{k}$

7.1.2 Quadratic membership function (Type-II). For each objective function, the corresponding quadratic membership functions are as follows:

$$
\mu_{F_{k}}=\left\{\begin{array}{lc}
1, & \text { if } F_{k} \leq F_{k}^{0}, \\
1-\left(\frac{F_{k}^{1}-F_{k}}{P_{k}}\right)^{2}, & \text { if } F_{k}^{0} \leq F_{k} \leq F_{k}^{1}, \\
0, & \text { if } \quad F_{k}^{1} \leq F_{k}
\end{array}\right.
$$

where $F_{k}^{0}$ and $F_{k}^{1}$ are to be chosen such that $F_{k}^{\text {min }} \leq F_{k}^{0} \leq F_{k}^{1} \leq$ $F_{k}^{\text {max }}$ and $P_{k}=F_{k}^{1}-F_{k}^{0}$ is the tolerance of k-th objective function $F_{k}$.

\subsection{Fuzzy decision method}

After determining the different linear/nnon-linear membership functions(MF) for each of the objective functions, following Bellman and Zadeh[1] and Zimmermannn[36], the given problem(10)can be formulated as

$$
\begin{gathered}
\text { Maximize } \\
\text { Subject to } \lambda \leq \mu_{F_{L}}, \quad \lambda \leq \mu_{F_{C}} \\
\\
0 \leq \lambda \leq 1 .
\end{gathered}
$$

with the help of two different types of membership functions given by (12) and (13),the above problem can be restated for a particular choice of DM as

$$
\begin{aligned}
& \text { Maximize } \quad \lambda \\
& \text { Subject to } \lambda \leq 1-\frac{F_{L}^{1}-F_{L}}{P_{L}}, \\
& \text { if the } M F \text { of first objective } \in \text { Type }-I \text {, } \\
& \lambda \leq 1-\left(\frac{F_{C}^{1}-F_{C}}{P_{C}}\right)^{2}, \\
& \text { if the } M F \text { of first objective } \in \text { Type }-I I, \\
& \quad 0 \leq \lambda \leq 1 \text {. }
\end{aligned}
$$

Here DM selects the above membership functions for the corresponding objective functions. Then the above problem can be solved by a non-linear optimization technique and optimal solution of $\lambda$, say $\lambda^{*}$ is obtained.

Now after obtaining $\lambda^{*}$, the DM selects the most important objective function from among the objective functions $F_{L}$ and $F_{C}$. Here $F_{L}$ is selected as DM would like to maximize his/her worst case. Then the problem becomes $\left(\lambda=\lambda^{*}\right)$

$$
\begin{array}{cc}
\text { Maximize } & \multicolumn{1}{c}{F_{L}} \\
\text { Subject to } & F_{L} \geq m_{L}, \quad F_{C} \geq m_{C} \\
& 0 \leq \lambda \leq 1 .
\end{array}
$$

$$
\begin{aligned}
& \text { Where } m_{L}=F_{L}^{1}-\left(F_{L}^{1}-F_{L}^{0}\right)\left(1-\lambda^{*}\right), \\
& \text { if the } M F \text { of first objective } \in \text { Type }-I, \\
& m_{C}=F_{C}^{1}-\left(F_{C}^{1}-F_{C}^{0}\right)\left(1-\lambda^{*}\right)^{\frac{1}{2}}, \\
& \text { if the } M F \text { of first objective } \in \text { Type }-I I, \\
& 0 \leq \lambda \leq 1 .
\end{aligned}
$$

\subsection{Pareto-optimal solution}

Now, after deriving the optimum decision variables, Paretooptimality test is performed according to Sakawa[31]. Let the decision variable $k^{*}$ and optimum values, $F_{L}^{*}=F_{L}\left(k^{*}\right)$ and $F_{C}^{*}=$ $F_{C}\left(k^{*}\right)$ are obtained from (16). With these values, the following problem is solving using a non-linear optimization technique:

$$
\begin{array}{r}
V=\left(\epsilon_{L}+\epsilon_{C}\right) \\
\text { Subject to } \quad F_{L}-\epsilon_{L}=F_{L}^{*}, \\
\\
F_{C}-\epsilon_{C}=F_{C}^{*} \\
\epsilon_{L}, \epsilon_{C} \geq 0, \quad 0 \leq \lambda \leq 1 .
\end{array}
$$

The optimal solutions of (17), say, $k, \bar{F}_{L}$ and $\bar{F}_{C}$ are called strong Pareto-optimal solutions of the problem(10) provided $V$ is very small; otherwise it is weak Pareto-optimal. 


\section{NUMERICAL EXAMPLE}

To illustrate the proposed inventory model, following crisp input data are considered in table-1. We consider case-I, when fuzzy parameters are TFN and case-II when fuzzy parameter are PFN.The nearest interval approximation according to Grzegorzewski[14] in both cases(i.e, case-I and case-II)are given in Table-2.

Following (14) and (15), the problem (9) is solved and the results are presented in Table- 3 and Table- 4 .

Let, with the above values, the membership functions of the objective functions be formed of the types as per Table 5 .

Let, at the beginning, analysis is performed to find optimum $\lambda$ with the membership functions $F_{L}$ as linear(Type-I) and $F_{C}$ as quadratic (Type-II). The optimum value of $\lambda$ is presented in Table-6.

With this value of $\lambda^{*}$, the objective function $F_{L}$ is optimized and the optimum results are given in Table-7.

Now, the results obtained from Table-7 are tested for paretooptimality and the pareto-optimal results are given in Table- 8 .

\section{DISCUSSION:}

In Table 8 , the values of $\mathrm{V}$ are quite small in both cases and hence, the optimum results in table 7 are strong Pareto-optimum and can be accepted.Still, if the decision-maker/practitioner is not satisfied with the outputs, he/she may perform the above analysis again re-choosing the membership functions for $F_{L}$ and $F_{C}$ as linear, quadratic and exponential(say). If the second time analysis does not also give the desired result, the DM may perform the analysis with the other possible different combinations(in this case, $3^{2}$ times) of the membership functions and can select the most suitable optimum solution for his/her firm/factory for implementation.Furthermore we observed that the average profit is greater in case-II than Case-I.

\section{CONCLUSION}

This production inventory model, which comprises of two production plants, two secondary warehouses and two showrooms, is aiming at minimizing the overall production cost per unit of product. In order to attain it we derived a closed form of solution of the model. Here, two plant concepts were used in the sense that a manufacturer / firm wanted to minimize the overall production cost by erecting two plants in two different regions / areas. In a nut shell, the study suggests that the production cost per unit of the product will minimize and thereby the total net profit will maximize by erecting two plants out of which one plant has substantially lower production cost per unit than the other.

Acknowledgements: Dr. Jayanta Kumar Dey thanks the Minor Research Project (PSW-138, 09/10 UGC, Govt. of India) for financial support to do this research work.

\section{REFERENCE}

[1] Bellman, R.E., and Zadeh, L.A; "Decision-making in a fuzzy environment", Management Science,Vol. 17, pp. B141B164,1970.

[2] Benkherouf,L; " A deterministic order level inventory model for deteriorating items with two storage facilities", International Journal Production Economics,vol. 48, pp. 167-175, 1997.

[3] Bhunia, A. K. and Maiti,M; " A two warehouse inventory model for a linear trend in demand", Opsearch, Vol. 31, pp. 318$329,1994$.
[4] Bhunia, A. K. and Maiti, M; "A deterministic two storage inventory model for variable production and inventory level dependent demand rate", Cahiers du CERO, Vol. 37, pp. 17-24, 1995.

[5] Bhunia,A. K., and Maiti, M; " A two warehouses inventory model for deteriorating items with a linear trend in demand and shortages", Journal of Operational Research Society,Vol. 49,pp. 289-292, 1998.

[6] Chung, K., Her, C., and Lin, S; " A two-warehouse inventory model with imperfect quality production processes", Computers and Industrial Engineering, Vol. 56,pp. 193-197, 2009.

[7] Chung, K. and Huang, T;" The optimal retailer's ordering policies for deteriorating items with limited storage capacity under trade credit financing". International Journal of Production Economics, Vol. 106, pp.127-146,2007.

[8] Dave, U; "On the EOQ models with two levels of storage", Opsearch, Vol. 25, pp. 190-196,1988.

[9] Dey, J.K., Kar,S., and Maiti,M;" An interactive method for inventory control with fuzzy lead-time and dynamic demand", European Journal of Operational Research, Vol. 167 ,pp. 381$397,2005$.

[10] Dey,J.K.,Mondal,S.K., and Maiti, M; " Two storage inventory problem with dynamic demand and interval valued leadtime over finite time horizon under inflation and time-value of money", European Journal of Operational Research, Vol. 185,pp. 170-194, 2008.

[11] Giri,B. C., Pal, S., Goswami, A., and Chaudhuri,K. S; "An inventory model for deteriorating items with stock-dependent demand rate", European Journal of Operational Research,Vol. 95, pp.604-610,1996.

[12] Giri, B. C., and Chaudhuri,K. S;'Deterministic models of perishable inventory with stock-dependent demand rate and nonlinear holding cost", European Journal of Operational Research, Vol. 105,pp. 67-474, 1998.

[13] Goswami,A. and Chaudhuri,K.S; " An economic order quantity model for items with two level of storage for a linear trend in demand", Journal of Operational Research Society,Vol.43, pp. 157-167, 1992.

[14] Grzegorzewski, P; "Nearest Interval approximation of a Fuzzy Number, Fuzzy Sets and Systems, Vol. 130,pp. 321330,2002 .

[15] Gupta, R. and Vrat P; " An EOQ model for stock dependent consumption rate",Opsearch,Vol. 23,pp. 19- 24,1986.

[16] Hariga, M;'Inventory models for multi-warehouse systems under fixed and flexible space leasing contracts". Computers and Industrial Engineering,Vol. 61,pp. 744-751,2011.

[17] Hartely,R. V;'Operations Research - A Managerial Emphasis, Good Year Publishing Company", California,pp. 315-317, 1976.

[18] Hsieh, T., Dye, C., and Ouyang, L; "Determining optimal lot size for a two-warehouse system with deterioration and shortages using net present value". European Journal of Operational research,Vol. 191, pp. 182-192, 2008.

[19] Kar,S. K., Bhunia, A. K., and Maiti, M; Deterministic inventory model with levels of storage, a linear trend in demand and a fixed time horizon, Computer and Operations ResearchVol. 28 , pp. 1315 - 1331, 2001.

[20] Lee, C. and Hsu, S; A two-warehouse production model for deteriorating inventory items with time dependent demands. European Journal of Operational Research,Vol. 194, pp. 700710,2009 . 
Table 1. Input data for different crisps parameter of the model.

\begin{tabular}{|c|c|c|c|c|c|c|c|c|c|c|c|c|c|c|}
\hline Parameter & $P_{1}$ & $P_{2}$ & $s_{1}$ & $s_{2}$ & $c_{p_{1}}$ & $c_{p_{2}}$ & $a_{1}$ & $b_{1}$ & $W_{1}$ & $a_{2}$ & $b_{2}$ & $W_{2}$ & $n$ & $r$ \\
\hline value & 100 & 200 & 90 & 70 & 75 & 50 & 110 & .5 & 75 & 75 & .5 & 50 & 11 & 18 \\
\hline
\end{tabular}

Table 2. Input data for different TFN/PFN parameter of the model.

\begin{tabular}{|c|c|c|c|}
\hline Parameter & Fuzzy value & $\begin{array}{c}\text { Case-I } \\
\text { Nearest interval for TFN }\end{array}$ & $\begin{array}{c}\text { Case-II } \\
\text { Nearest } \text { interval for PFN }\end{array}$ \\
\hline 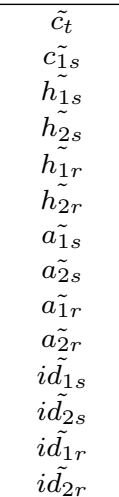 & $\begin{array}{c}{[24,30,36]} \\
{[.04, .1, .16]} \\
{[.24, .3, .36]} \\
{[.14, .2, .26]} \\
{[.14, .2, .26]} \\
{[.04, .1, .16]} \\
{[380,400,420]} \\
{[280,300,320]} \\
{[180,200,220]} \\
{[80,100,120]} \\
{[.4, .6, .8]} \\
{[.1, .3, .5]} \\
{[.16, .2, .26]} \\
{[.04, .1, .16]}\end{array}$ & $\begin{array}{c}{\left[c_{t L}, c_{t R}\right]=[27,33]} \\
{\left[c_{s_{1 L}}, c_{s_{1 R}}\right]=[.07, .13]} \\
{\left[h_{1 s_{L}}, h_{1 s_{R}}\right]=[.27, .33]} \\
{\left[h_{2 s_{L}}, h_{2 s_{R}}\right]=[.17, .23]} \\
{\left[h_{1 r_{L}}, h_{1 r_{R}}\right]=[.17, .23]} \\
{\left[h_{2 r_{L}}, h_{2 r_{R}}\right]=[.07, .13]} \\
{\left[a_{1 s_{L}}, a_{1 s_{R}}\right]=[390,410]} \\
{\left[a_{2 s_{L}}, a_{2 s_{R}}\right]=[290.310]} \\
{\left[a_{1 r_{L}}, a_{1 r_{R}}\right]=[190,210]} \\
{\left[a_{2 r_{L}}, a_{2 r_{R}}\right]=[90,110]} \\
{\left[i d_{1 s_{L}}, i d_{1 s_{R}}\right]=[.5, .7]} \\
{\left[i d_{2 s_{L}}, i d_{2 s_{R}}\right]=[.2, .4]} \\
{\left[i d_{1 r_{L}}, i d_{1 r_{R}}\right]=[.18, .23]} \\
{\left[i d_{2 r_{L}}, i d_{2 r_{R}}\right]=[.07, .13]}\end{array}$ & $\begin{array}{c}{\left[c_{t L}, c_{t R}\right]=[25.66,32]} \\
{\left[c_{s_{1 L}}, c_{s_{1 R}}\right]=[.06, .12]} \\
{\left[h_{1 s_{L}}, h_{1 s_{R}}\right]=[.256, .32]} \\
{\left[h_{2 s_{L}}, h_{2 s_{R}}\right]=[.16, .22]} \\
{\left[h_{1 r_{L}}, h_{1 r_{R}}\right]=[.16, .22]} \\
{\left[h_{2 r_{L}}, h_{2 r_{R}}\right]=[.06, .12]} \\
{\left[a_{1 s_{L}}, a_{1 s_{R}}\right]=[386.66,406.66]} \\
{\left[a_{2 s_{L}}, a_{2 s_{R}}\right]=[286.66,306.66]} \\
{\left[a_{1 r_{L}}, a_{1 r_{R}}\right]=[186.66,206.66]} \\
{\left[a_{2 r_{L}}, a_{2 r_{R}}\right]=[86.66,106.66]} \\
{\left[i d_{1 s_{L}}, i d_{1 s_{R}}\right]=[.46, .66]} \\
{\left[i d_{2 s_{L}}, i d_{2 s_{R}}\right]=[.16, .36]} \\
{\left[i d_{1 r_{L}}, i d_{1 r_{R}}\right]=[.17, .22]} \\
{\left[i d_{2 r_{L}}, i d_{2 r_{R}}\right]=[.06, .12]}\end{array}$ \\
\hline
\end{tabular}

[21] Lee, C. and Ma, C;'O'Optimal inventory policy for deteriorating items with two-warehouse and time-dependent demands".Production Planning and Control,Vol. 11, pp. 689-696, 2000.

[22] Levin, R. I., Mclaughlin, C. P. , Lamone, R.P. and Kottas, J. F; "Production / Operations management: contemporary policy for managing operating systems", McGraw-Hill, New York,pp. 373-387,1972.

[23] Liang, Y. and Zhou, F;"A two-warehouse inventory model for deteriorating items under conditionally permissible delay in payment”. Applied Mathematical Modelling, Vol.35,pp. 22212231,2011 .

[24] Liao, J. and Huang, K; "Deterministic inventory model for deteriorating items with trade credit financing and capacity constraints". Computers and Industrial Engineering, Vol. 59,pp. 611-618, 2010.

[25] Mandal, B. N. and Phaujder, S; "An inventory model for deteriorating items and stock dependent consumption rate",Journal of Oprational Research Society,Vol. 40, pp. 483 - 488, 1989A.

[26] Mandal, B. N. and Phaujder, S;"A note on an inventory model with stock dependent consumption rate", Opsearch,Vol. 26,pp. 43-46, 1989.

[27] Niu, B. and Xie, J; "A note on "two-warehouse inventory model with deterioration under FIFO dispatch policy", European Journal of Operational Research, Vol.190, pp. 571-577,2008.

[28] Padmanabhan, G. , and Vrat,P;" EOQ models for perishable items under stock-dependent selling rate", European Journal of Operational Research,Vol. 86,pp. 281-292, 1995.

[29] Pakkala, T. P. M. and Achary, K. K;"A deterministic inventory model for deteriorating items with two warehouses and finite replenishment rate",European Journal of Operational Research, Vol. 57, pp. 71-76,1992.

[30] Pal,S., Goswami, A., and Chaudhuri, K. S;" A deterministic inventory model for deteriorating items with stock-dependent demand rate", International Journal Production Economics, Vol. 32 , pp. 291-299, 1993.
[31] Sakawa,M;'Interactive fuzzy decision making for multiobjective linear programming problems and application,in: E. sanche(Ed.)", Proceedings of IFAC Symposium of Fuzzy Information, Knowledge Representation and Decision Analysis,pp. 293-298, July 1983.

[32] Sarkar,B. R., Mukherjee,S., and Balan,C. V; "An order-level lot-size inventory model with inventory-level dependent demand and deterioration",International Journal Production Economics, Vol. 48 ,pp. 227-236, 1997.

[33] Sarma, K. V. S; "A deterministic inventory model with two levels of storage and an optimum release rule", Opsearch, Vol. 20,pp. 175-180, 1983.

[34] Urban, T. C; " Deterministic inventory models incorporating marketing decisions", Computers and Industrial Engineering, Vol. 22,pp. 85-93, 1992A.

[35] Urban,T. L; "An inventory model with an inventory-leveldependent demand rate and relaxed terminal conditions",Journal of Operational Research Society, Vol. 43,pp. 721-724, 1992B.

[36] Zimmermann, H.Z; "Fuzzy linear programing with several objective functions",Fuzzy Set and Systems, Vol. 1, pp. 46-55, 1978.

[37] Zhou,Y. W;"An optimal EOQ model for deteriorating items with two warehouses and time varying demand", Mathematica Applicata, Vol. 10, pp. $19-23,1998$.

[38] Zhou, Y. and Yang, S;" A two-warehouse inventory model for items with stock-level-dependent demand rate", International Journal of Production Economics, Vol. 95, pp. 215- 228,2005.

\subsection{Appendix- $A$}

$$
\begin{aligned}
F_{L} & =\frac{X_{L} k^{2}+Y_{L} k+Y_{L}}{U+V k} \\
\text { and, } F_{R} & =\frac{X_{R} k^{2}+Y_{R} k+Y_{R}}{U+V k}
\end{aligned}
$$


Table 3. Individual minimum and maximum of objective function.

\begin{tabular}{|c|c|c|c|}
\hline cases & Case $-I$ & & Case - II \\
\hline Objective functions & Minimum & Maximum & Minimum \\
\hline$F_{L}$ & 3561.014 & 3622.093 & 3642.440 \\
$F_{C}$ & 3807.755 & 3881.665 & 3905.213 \\
\hline
\end{tabular}

Table 4. Input data for $F_{k}^{0}, F_{k}^{1}$.

\begin{tabular}{|c|c|c|c|c|}
\hline cases & Case-I & & Case-II & \\
\hline Objective functions & $F_{k}^{0}$ & $F_{k}^{1}$ & $F_{k}^{0}$ & $F_{k}^{1}$ \\
\hline $\begin{array}{l}F_{L} \\
F_{C}\end{array}$ & $\begin{array}{l}3561.015 \\
3807.756\end{array}$ & $\begin{array}{l}3622.092 \\
3881.664\end{array}$ & $\begin{array}{l}3642.441 \\
3905.214\end{array}$ & $\begin{array}{l}3707.426 \\
3983.939\end{array}$ \\
\hline
\end{tabular}

where $X_{L}, X_{R}, Y_{L}, Y_{R}, Z_{L}$ and $Z_{R}$ are given by

$$
\begin{aligned}
X_{L}= & -\left[c_{1 s_{R}}\left(a_{1}-p_{1}\right)\left[\frac{B p_{1}^{\prime}}{\left(P_{2}-a_{2}-b_{2} W_{2}\right)^{2}}\right] \frac{p_{1}^{\prime}}{P_{2}-a_{2}-b_{2} W_{2}}\right. \\
+ & c_{1 s_{R}}\left(a_{1}-p_{1}\right)\left[\frac{B^{2}}{\left(P_{2}-a_{2}-b_{2} W_{2}\right)^{2}}\right]+h_{1_{r R}} \frac{B}{P_{2}-a_{2}-b_{2} W_{2}} \\
& \quad\left[n P_{2} \frac{p_{1}^{\prime}}{p_{1}^{\prime \prime}}-\left(a_{2}+b_{2} W_{2}\right)(m-r)\right]+h_{1_{r R}}\left[\frac{n}{2} p_{1}^{\prime}+\frac{m+1}{2} \frac{p_{1}^{\prime 2}}{p_{1}^{\prime \prime}}\right] \\
+ & h_{2_{r R}}\left[\frac{p_{1}^{\prime 2}}{2\left(P_{2}-a_{2}-b_{2} W_{2}\right)}+\frac{P_{2}-a_{2}-b_{2} W_{2}}{2}\left(n^{2}+r^{2}\right.\right. \\
+ & \left.\frac{2 n r p_{1}^{\prime}}{P_{1}^{\prime}}\right)-\frac{n(n-1)}{2} p_{1}^{\prime}-r(n+r-1) \frac{p_{1}^{\prime 2}}{p_{1}^{\prime \prime}}+n P_{2}\left(n \frac{p_{1}^{\prime}}{p_{1}^{\prime \prime}}+r \frac{p_{1}^{\prime 2}}{p_{1}^{\prime 2}}\right) \\
- & \frac{a_{2}+b_{2} W_{2}}{2}\left((m-r)^{2}+2(m-r)\left(n \frac{p_{1}^{\prime}}{p_{1}^{\prime \prime}}+r \frac{p_{1}^{\prime 2}}{p_{1}^{\prime \prime 2}}\right)\right. \\
- & \left.\frac{(m-r)(m+n-1)}{2} p_{1}^{\prime 2}\right) \frac{p_{1}^{\prime}}{P_{2}-a_{2}-b_{2} W_{2}}\left(n P_{2} \frac{p_{1}^{\prime}}{p_{1}^{\prime \prime}}\right. \\
- & \left.\left.\left.\left(a_{2}+b_{2} W_{2}\right)(m-r)\right)\right]+c_{1 s_{R}} \frac{\left(a_{1}-P_{1}\right) p_{1}^{\prime 2}}{2\left(P_{2}-a_{2}-b_{2} W_{2}\right)^{2}}\right],
\end{aligned}
$$$$
X_{R}=-\left[c_{1 s_{L}}\left(a_{1}-p_{1}\right)\left[\frac{B p_{1}^{\prime}}{\left(P_{2}-a_{2}-b_{2} W_{2}\right)^{2}}\right] \frac{p_{1}^{\prime}}{P_{2}-a_{2}-b_{2} W_{2}}\right.
$$$$
+c_{1 s_{L}}\left(a_{1}-p_{1}\right)\left[\frac{B^{2}}{\left(P_{2}-a_{2}-b_{2} W_{2}\right)^{2}}\right]+h_{1_{r L}} \frac{B}{P_{2}-a_{2}-b_{2} W_{2}}
$$$$
\left[n P_{2} \frac{p_{1}^{\prime}}{p_{1}^{\prime \prime}}-\left(a_{2}+b_{2} W_{2}\right)(m-r)\right]+h_{1_{r L}}\left[\frac{n}{2} p_{1}^{\prime}+\frac{m+1}{2} \frac{p_{1}^{\prime 2}}{p_{1}^{\prime \prime}}\right]
$$$$
+h_{2_{r L}}\left[\frac{p_{1}^{\prime 2}}{2\left(P_{2}-a_{2}-b_{2} W_{2}\right)}+\frac{P_{2}-a_{2}-b_{2} W_{2}}{2}\left(n^{2}+r^{2}\right.\right.
$$$$
\left.+\frac{2 n r p_{1}^{\prime}}{p_{1}^{\prime}}\right)-\frac{n(n-1)}{2} p_{1}^{\prime}-r(n+r-1) \frac{p_{1}^{\prime 2}}{p_{1}^{\prime \prime}}+n P_{2}\left(n \frac{p_{1}^{\prime}}{p_{1}^{\prime \prime}}\right.
$$$$
\left.+r \frac{p_{1}^{\prime 2}}{p_{1}^{\prime \prime 2}}\right)-\frac{a_{2}+b_{2} W_{2}}{2}\left((m-r)^{2}+2(m-r)\left(n \frac{p_{1}^{\prime}}{p_{1}^{\prime \prime}}+r \frac{p_{1}^{\prime 2}}{p_{1}^{\prime \prime 2}}\right)\right.
$$$$
\left.-\frac{(m-r)(m+n-1)}{2} p_{1}^{\prime 2}\right) \frac{p_{1}^{\prime}}{P_{2}-a_{2}-b_{2} W_{2}}\left(n P_{2} \frac{p_{1}^{\prime}}{p_{1}^{\prime \prime}}\right.
$$$$
\left.\left.\left.-\left(a_{2}+b_{2} W_{2}\right)(m-r)\right)\right]-c_{1 s_{L}} \frac{\left(a_{1}-P_{1}\right) p_{1}^{\prime 2}}{2\left(P_{2}-a_{2}-b_{2} W_{2}\right)^{2}}\right],
$$

$Y_{L}=\left(s_{1}-s_{2}\right) B+\left[\frac{B}{p_{2}-a_{2}-b_{2} W_{2}}\right]\left[\left(s_{1}-c_{p_{1}}\right) P_{1}\right.$

$$
\left.+\left(s_{2}-c_{p_{2}}\right) P_{2}\right]-2 c_{1 s_{R}}\left(a_{1}-P_{1}\right) B\left[\left(P_{2}-a_{2}-b_{2} W_{2}\right) t_{1}^{\prime}\right.
$$$$
\left.+\frac{A+W_{1}}{\left(p_{2}-a_{2}-b_{2} W_{2}\right)^{2}}\right]+\left(n+\frac{p_{1}^{\prime}}{P_{2}-a_{2}-b_{2} W_{2}}\right)
$$$$
\left[\left(s_{1}-c_{p_{1}}\right) P_{1}+\left(s_{2}-c_{p_{2}}\right) P_{2}\right]+r\left(s_{2}-c_{p_{1}}\right) P_{2} \frac{p_{1}^{\prime}}{p_{1}^{\prime \prime}}
$$$$
+\left(s_{1}-s_{2}\right)(m+n+1) p_{1}^{\prime}-c_{1 s_{R}}\left(a_{1}-p_{1}\right)\left[t_{1}^{\prime}\right.
$$$$
\left.+\frac{A+W_{1}}{P_{2}-a_{2}-b_{2} W_{2}}\right] \frac{p_{1}^{\prime}}{P_{2}-a_{2}-b_{2} W_{2}}-h_{1 s R} W_{1}[(n+1)
$$$$
\left.+\frac{m P_{1}^{\prime}}{p_{1}^{\prime \prime}}\right]-h_{2 s R}\left[W_{2}\left(1+\frac{P_{2}}{a_{2}-b_{2} W_{2}}\right)\left(\frac{B}{P_{2}-a_{2}-b_{2} W_{2}}\right)\right.
$$$$
\left.-\frac{a_{2}}{b_{2}^{2}} \log \left(1+\frac{b_{2} W_{2}}{a_{2}}\right)\right]-h_{2 s R} W_{2}\left[n+\frac{p_{1}^{\prime}}{P_{2}-a_{2}-b_{2} W_{2}}+\frac{m p_{1}^{\prime}}{p_{1}^{\prime \prime}}\right.
$$$$
\left.+\frac{P_{2}}{a_{2}+b_{2} W_{2}}\left(n+\frac{p_{1}^{\prime}}{P_{2}-a_{2}-b_{2} W_{2}}+\frac{r p_{1}^{\prime}}{p_{1}^{\prime \prime}}\right)-\frac{(m+n) p_{1}^{\prime}}{a_{2}-b_{2} W_{2}}\right]
$$$$
-h_{1 r R}\left(t_{1}^{\prime}+\frac{A+W_{1}}{P_{2}-a_{2}-b_{2} W_{2}}\right)\left[n P_{2} \frac{p_{1}^{\prime}}{p_{1}^{\prime \prime}}-\left(a_{2}+b_{2} W_{2}\right)(m-r)\right]
$$$$
-h_{2 r R} \frac{P_{2}}{2}\left[n+\frac{p_{1}^{\prime}}{P_{2}-a_{2}-b_{2} W_{2}}+\frac{r p_{1}^{\prime}}{p_{1}^{\prime \prime}}-\frac{m+n}{2} p_{1}^{\prime}\right]
$$$$
-i d_{1 s R} \frac{r p_{1}^{\prime}}{p_{1}^{\prime \prime}}-\left(i d_{1 r R}+h_{2 r R} \frac{P_{2}}{2}\right)\left(\frac{B}{P_{2}-a_{2}-b_{2} W_{2}}\right)-i d_{1 r R}
$$$$
\left[\frac{p_{1}^{\prime}}{P_{2}-a_{2}-b_{2} W_{2}}-\frac{(m-r+1) p_{1}^{\prime}}{p_{1}^{\prime \prime}}\right]-c_{t R}(m+m+1) p_{1}^{\prime}
$$$$
Y_{R}=\left(s_{1}-s_{2}\right) B+\left[\frac{B}{p_{2}-a_{2}-b_{2} W_{2}}\right]\left[\left(s_{1}-c_{p_{1}}\right) P_{1}\right.
$$$$
\left.+\left(s_{2}-c_{p_{2}}\right) P_{2}\right]-2 c_{1 s_{L}}\left(a_{1}-P_{1}\right) B\left[\left(P_{2}-a_{2}-b_{2} W_{2}\right) t_{1}^{\prime}\right.
$$$$
\left.+\frac{A+W_{1}}{\left(P_{2}-a_{2}-b_{2} W_{2}\right)^{2}}\right]+\left(n+\frac{p_{1}^{\prime}}{P_{2}-a_{2}-b_{2} W_{2}}\right)
$$$$
\left[\left(s_{1}-c_{p_{1}}\right) P_{1}+\left(s_{2}-c_{p_{2}}\right) P_{2}\right]+r\left(s_{2}-c_{p_{1}}\right) P_{2} \frac{p_{1}^{\prime}}{p_{1}^{\prime \prime}}
$$$$
+\left(s_{1}-s_{2}\right)(m+n+1) p_{1}^{\prime}-c_{1 s_{L}}\left(a_{1}-p_{1}\right)\left[t_{1}^{\prime}\right.
$$$$
\left.+\frac{A+W_{1}}{P_{2}-a_{2}-b_{2} W_{2}}\right] \frac{p_{1}^{\prime}}{P_{2}-a_{2}-b_{2} W_{2}}
$$$$
-h_{1 s L} W_{1}\left[(n+1)+\frac{m p_{1}^{\prime}}{p_{1}^{\prime \prime}}\right]-h_{2 s L}\left[W_{2}\left(1+\frac{P_{2}}{a_{2}-b_{2} W_{2}}\right)\right.
$$ 


$$
\begin{aligned}
& \left.\left(\frac{B}{P_{2}-a_{2}-b_{2} W_{2}}\right)-\frac{a_{2}}{b_{2}^{2}} \log \left(1+\frac{b_{2} W_{2}}{a_{2}}\right)\right]-h_{2 s L} W_{2} \\
& {\left[n+\frac{p_{1}^{\prime}}{P_{2}-a_{2}-b_{2} W_{2}}+\frac{m p_{1}^{\prime}}{p_{1}^{\prime \prime}}+\frac{P_{2}}{a_{2}+b_{2} W_{2}}(n\right.} \\
& \left.\left.+\frac{p_{1}^{\prime}}{P_{2}-a_{2}-b_{2} W_{2}}+\frac{r p_{1}^{\prime}}{p_{1}^{\prime \prime}}\right)-\frac{(m+n) p_{1}^{\prime}}{a_{2}-b_{2} W_{2}}\right] \\
& -h_{1 r L}\left(t_{1}^{\prime}+\frac{A+W_{1}}{P_{2}-a_{2}-b_{2} W_{2}}\right)\left[n P_{2} \frac{p_{1}^{\prime}}{p_{1}^{\prime \prime}}-\left(a_{2}+b_{2} W_{2}\right)\right. \\
& (m-r)]-h_{2 r L} \frac{P_{2}}{2}\left[n+\frac{p_{1}^{\prime}}{P_{2}-a_{2}-b_{2} W_{2}}+\frac{r p_{1}^{\prime}}{p_{1}^{\prime \prime}}\right. \\
& \left.-\frac{m+n}{2} p_{1}^{\prime}\right]-i d_{1 s L} \frac{r p_{1}^{\prime}}{p_{1}^{\prime \prime}}-\left(i d_{1 r L}+h_{2 r L} \frac{P_{2}}{2}\right) \\
& \left(\frac{B}{P_{2}-a_{2}-b_{2} W_{2}}\right)-i d_{1 r L}\left[\frac{p_{1}^{\prime}}{P_{2}-a_{2}-b_{2} W_{2}}\right. \\
& \left.-\frac{(m-r+1) p_{1}^{\prime}}{p_{1}^{\prime \prime}}\right]-c_{t L}(m+m+1) p_{1}^{\prime} \text {, } \\
& Z_{L}=\left(s_{1}-s_{2}\right)\left(A+W_{1}\right)+\left[t_{1}^{\prime}+\frac{A+W_{1}}{P_{2}-a_{2}-b_{2} W_{2}}\right] \\
& {\left[\left(s_{1}-c_{p_{1}}\right) P_{1}+\left(s_{2}-c_{p_{2}}\right) P_{2}\right]-c_{1 s_{R}}\left(a_{1}-P_{1}\right)} \\
& {\left[t_{1}^{\prime}+\frac{A+W_{1}}{P_{2}-a_{2}-b_{2} W_{2}}\right]^{2}-c_{1 s_{R}}\left(a_{1}-P_{1}\right)} \\
& {\left[\frac{\left(P_{2}-a_{2}-b_{2} W_{2}\right) t_{1}^{\prime}+A+W_{1}}{P_{2}-a_{2}-b_{2} W_{2}}\right]^{2}-h_{1 s R}\left[\frac{W_{1}}{b_{1}}\right.} \\
& \left.-\frac{a_{1}}{b_{1}^{2}} \log \left(1+\frac{b_{1} W_{1}}{a_{1}}\right)\right]-h_{2 s R}\left[\frac { P _ { 2 } - a _ { 2 } } { b _ { 2 } } \left(t_{1}^{\prime}\right.\right. \\
& \left.+\frac{1}{b_{2}} \frac{P_{2}-a_{2}-b_{2} W_{2}}{P_{2}-a_{2}}\right)+W_{2}\left(1+\frac{P_{2}}{a_{2}-b_{2} W_{2}}\right) \\
& \left.\left(t_{1}^{\prime}+\frac{A+W_{1}}{P_{2}-a_{2}-b_{2} W_{2}}\right)-\frac{a_{2}}{b_{2}^{2}} \log \left(1+\frac{b_{2} W_{2}}{a_{2}}\right)\right] \\
& -\left(i d_{1 r R}+h_{2 r R} \frac{P_{2}}{2}\right)\left(t_{1}^{\prime}+\frac{A+W_{1}}{P_{2}-a_{2}-b_{2} W_{2}}\right) \\
& -i d_{2 r R}\left[\frac{1}{b_{2}} \log \left(1+\frac{b_{2} W_{2}}{a_{2}}\right)-c_{t R}\left(A+W_{1}\right)\right. \\
& \text { - } \left.A_{1 s R}-A_{2 s R}-A_{1 r R}-A_{2 r R}\right] \\
& \text { and, } Z_{R}=\left(s_{1}-s_{2}\right)\left(A+W_{1}\right)+\left[t_{1}^{\prime}+\frac{A+W_{1}}{p_{2}-a_{2}-b_{2} W_{2}}\right] \\
& {\left[\left(s_{1}-c_{p_{1}}\right) P_{1}+\left(s_{2}-c_{p_{2}}\right) P_{2}\right]-c_{1 s_{L}}\left(a_{1}-P_{1}\right)} \\
& {\left[t_{1}^{\prime}+\frac{A+W_{1}}{P_{2}-a_{2}-b_{2} W_{2}}\right]^{2}-c_{1 s_{L}}\left(a_{1}-P_{1}\right)} \\
& {\left[\frac{\left(P_{2}-a_{2}-b_{2} W_{2}\right) t_{1}^{\prime}+A+W_{1}}{P_{2}-a_{2}-b_{2} W_{2}}\right]^{2}-h_{1 s L}\left[\frac{W_{1}}{b_{1}}\right.} \\
& \left.-\frac{a_{1}}{b_{1}^{2}} \log \left(1+\frac{b_{1} W_{1}}{a_{1}}\right)\right]-h_{2 s L}\left[\frac { P _ { 2 } - a _ { 2 } } { b _ { 2 } } \left(t_{1}^{\prime}\right.\right. \\
& \left.+\frac{1}{b_{2}} \frac{P_{2}-a_{2}-b_{2} W_{2}}{P_{2}-a_{2}}\right)+W_{2}\left(1+\frac{P_{2}}{a_{2}-b_{2} W_{2}}\right) \\
& \left.\left(t_{1}^{\prime}+\frac{A+W_{1}}{P_{2}-a_{2}-b_{2} W_{2}}\right)-\frac{a_{2}}{b_{2}^{2}} \log \left(1+\frac{b_{2} W_{2}}{a_{2}}\right)\right]
\end{aligned}
$$$$
-\left(i d_{1 r L}+h_{2 r L} \frac{P_{2}}{2}\right)\left(t_{1}^{\prime}+\frac{A+W_{1}}{P_{2}-a_{2}-b_{2} W_{2}}\right)
$$$$
\text { - } i d_{2 r L}\left[\frac{1}{b_{2}} \log \left(1+\frac{b_{2} W_{2}}{a_{2}}\right)-c_{t L}\left(A+W_{1}\right)\right.
$$$$
\text { - } \left.A_{1 s L}-A_{2 s L}-A_{1 r L}-A_{2 r L}\right]
$$ 
Table-5. Types of MF for objective functions.

\begin{tabular}{|c|c|}
\hline Objective function & Types of membership functions \\
\hline$F_{L}$ & Types $-I$ \\
$F_{C}$ & Types $-I I$ \\
\hline
\end{tabular}

Table-6: Optimal values of $\lambda$

\begin{tabular}{|c|c|c|}
\hline Maximum $\lambda$ & Case $-I$ & Case - II \\
\hline$\lambda^{*}$ & .9975313 & .9973593 \\
\hline
\end{tabular}

Table-7: Optimal results when $F_{L}$ is chosen as the most important objective function

\begin{tabular}{|c|c|c|c|c|}
\hline Parameter & $k^{*}$ & $F_{L}^{*}$ & $F_{C}^{*}$ & $F_{R}^{*}$ \\
\hline Case-I & 1.084373 & 3621.941 & 3877.992 & 3922.341 \\
Case-II & 1.172279 & 3707.254 & 3979.894 & 4023.375 \\
\hline
\end{tabular}

Table-8: Pareto-optimal results

\begin{tabular}{|c|c|c|c|c|}
\hline Parameter & $k^{*}$ & $F_{L}^{*}$ & $F_{C}^{*}$ & $V^{*}$ \\
\hline Case-I & 1.084409 & 3621.942 & 3877.993 & .000857474 \\
Case-II & 1.172386 & 3707.255 & 3979.897 & .000847669 \\
\hline
\end{tabular}

